\title{
Vortex-vortex interaction in bulk superconductors: Ginzburg-Landau theory
}

\author{
Andrey Chaves, ${ }^{1,2, *}$ F. M. Peeters, ${ }^{1,2}$ G. A. Farias, ${ }^{2, \dagger}$ and M. V. Milošević ${ }^{1}$ \\ ${ }^{1}$ Department of Physics, University of Antwerp, Groenenborgerlaan 171, B-2020 Antwerp, Belgium \\ ${ }^{2}$ Departamento de Física, Universidade Federal do Ceará, Caixa Postal 6030, Campus do Pici, 60455-900 Fortaleza, Ceará, Brazil
}

(Received 21 May 2010; published 24 February 2011; corrected 21 March 2011)

\begin{abstract}
The vortex-vortex interaction potential in bulk superconductors is calculated within the Ginzburg-Landau (GL) theory and is obtained from a numerical solution of a set of two coupled nonlinear GL differential equations for the vector potential and the superconducting order parameter, where the merger of vortices into a giant vortex is allowed. Further, the interaction potentials between a vortex and a giant vortex and between a vortex and an antivortex are obtained for both type-I and type-II superconductors. Our numerical results agree asymptotically with the analytical expressions for large intervortex separations that are available in the literature. We propose empirical expressions valid over the full interaction range, which are fitted to our numerical data for different values of the GL parameter.
\end{abstract}

DOI: 10.1103/PhysRevB.83.054516

PACS number(s): 74.20.De, 74.25.Wx

\section{INTRODUCTION}

The interaction potential between vortices has been an important subject of study for many years. In 1971, Kramer ${ }^{1}$ used the asymptotic behavior of the vortex fields for large distances in the Abelian Higgs model to obtain an analytical expression for the vortex-vortex interaction potential, which is given by modified Bessel functions. This potential is attractive (repulsive) for type-I (type-II) systems, i.e., for a superconductor Ginzburg-Landau (GL) parameter with $\kappa=\lambda / \xi<1 / \sqrt{2}(>1 / \sqrt{2})$, where $\lambda$ is the penetration depth and $\xi$ is the coherence length. Moreover, this expression leads to a constant interaction energy as a function of the separation between vortices for the critical value $\kappa=1 / \sqrt{2}$ (also called the Bogomol'nyi point), implying that vortices do not interact in this regime. A detailed analysis of the vortex-vortex $(\mathrm{V}-\mathrm{V})$ interaction was given later by Jacobs and Rebbi, ${ }^{2}$ who constructed a variational function describing two separate vortices and obtained the variational parameters by minimizing the free energy. This variational function was able to model (i) the deformation of the vortex core when the vortices are brought close to each other, and (ii) the formation of a giant vortex ${ }^{3-5}$ when vortices are superimposed on each other.

Thereafter, several works studied different aspects of the interaction between vortices in superconductors. For example, Brandt ${ }^{6}$ constructed an effective interaction potential between vortex lines such that it reproduces the full nonlocal elastic energy of flux-line lattices in type-II superconductors. This potential correctly vanishes and changes sign at $\kappa=1 / \sqrt{2}$. Speight $^{7}$ derived the V-V interaction from a linear field theory described by a Lagrangian of two singular point sources placed at the vortex centers. MacKenzie et al. ${ }^{8}$ used the linear field theory proposed by Speight to obtain the interaction between separated strings in a model with two order parameters, which may be relevant for superconducting cosmic strings, ${ }^{9}$ the $\mathrm{SO}(5)$ model of high-temperature superconductivity, and solitons in nonlinear optics. Eventually, all these analytical approximations lead to expressions that are the same, or at least very similar, to the one derived by Kramer for large separation between vortices. Other models were presented by Mohamed et al. ${ }^{10}$ who used a perturbative approach to calculate the $\mathrm{V}-\mathrm{V}$ interaction for superconductors with $\kappa \approx 1 / \sqrt{2}$ when the GL theory is extended to low temperatures, and by Hernández and López, ${ }^{11}$ who used a variational approach based on the Clem trial function ${ }^{12}$ to calculate the force between vortices. Auzzi et al. ${ }^{13}$ showed recently that for non-Abelian vortex interactions, there are two other regimes besides the well-known type-I and type-II interactions: Depending on the relative orientation, the interaction potential can show attractive and repulsive regions for the same system. Similar behavior can be achieved in a two-band superconductor. ${ }^{14,15}$

In the present work, we solve numerically the set of GL differential equations for two vortices fixed at a certain distance from each other. We are able to recover the V-V potential obtained by Jacobs and Rebbi ${ }^{2}$ and the asymptotic behaviors predicted by Kramer. ${ }^{1}$ The results are generalized to the case of vortex-giant vortex (V-GV) and vortex-antivortex (V-AV) interactions. We parametrize all obtained forces for future use in, e.g., molecular-dynamics (MD) simulations of the vortex matter.

The remainder of this paper is organized as follows: In Sec. II, we review the asymptotic behavior of the magnetic field and order parameter of the single static vortex in the GL theory and expand the analytical expression suggested by Kramer to study the interaction between a single vortex and another vortex, an antivortex or a giant vortex, in the limit of large separation between them. In Sec. III we discuss the set of coupled nonlinear differential equations that describe these interactions, which are valid for arbitrary separation between vortices, and thus also for small distances where deformations of the interacting vortex cores are important. This set of equations is solved numerically without any approximations for arbitrary values of $\kappa$, and the results for the V-V, V-GV, and $\mathrm{V}-\mathrm{AV}$ interactions are shown in Sec. IV and compared to the analytical expressions obtained in Sec. II. For each of these cases, a fitting function for the interaction is proposed and the fitting parameters are given. Our results are summarized in Sec. V.

\section{ASYMPTOTICS OF THE INTERVORTEX POTENTIAL}

Let us start with the expression for the free energy in the GL theory, or equivalently, the potential in the Abelian Higgs 
model: $:^{2,14}$

$$
E=\int \mathcal{F} d \overrightarrow{\mathrm{r}}
$$

where the functional $\mathcal{F}$ is given by

$\mathcal{F}=\frac{\hbar^{2}}{4 m}\left|\left(\vec{\nabla}-i \frac{2 e}{\hbar c} \overrightarrow{\mathrm{A}}\right) \psi\right|^{2}+\frac{1}{8 \pi}|\vec{\nabla} \times \overrightarrow{\mathrm{A}}|^{2}+\frac{\beta}{2}|\psi|^{4}-\alpha|\psi|^{2}$.

In this expression, $\psi$ is the order parameter (a complex scalar field), $\vec{A}$ is the electromagnetic vector (gauge) potential, and $\alpha$ and $\beta$ are phenomenological parameters, which are related to the two characteristic lengths in a superconductor: the coherence length $\xi=\hbar / \sqrt{4 m \alpha}$ and the penetration depth $\lambda=c / e(\sqrt{m \beta / 8 \pi \alpha})$. It is convenient to define $\mu=\sqrt{2} \kappa$, so that when $\mu<1(\mu>1)$ we are in the type-I (type-II) regime $(\kappa=1 / \sqrt{2}$ leads to $\mu=1){ }^{2}$ If we define $\vec{A}=(2 e / \hbar c) \overrightarrow{\mathrm{A}}$ and $\Psi=\sqrt{\beta / \alpha} \psi / \lambda$, the expression for the energy functional can be rewritten in dimensionless units as

$$
F=\frac{1}{2}|(\vec{\nabla}-i \vec{A}) \Psi|^{2}+\frac{1}{2}|\vec{\nabla} \times \vec{A}|^{2}+\frac{\mu^{2}}{8}\left(1-|\Psi|^{2}\right)^{2},
$$

where the distances are scaled by the magnetic-field penetration depth $\lambda$, energy by $E_{0}=\beta / 2 \alpha^{2} \xi^{2}$, and the force by $\Omega_{0}=\beta / 2 \alpha^{2} \xi^{2} \lambda$.

The lowest-energy configuration of the system is found by minimizing $E$ with respect to $\Psi$ and the vector potential $\vec{A}$. The standard way of minimizing a functional is by the Euler-Lagrange equations, which in the case of Eq. (1) are

$$
\frac{\partial F}{\partial \Psi}-\sum_{i} \frac{\partial}{\partial x_{i}} \frac{\partial F}{\partial\left(\frac{\partial \Psi}{\partial x_{i}}\right)}=0
$$

and

$$
\frac{\partial F}{\partial A_{j}}-\sum_{i} \frac{\partial}{\partial x_{i}} \frac{\partial F}{\partial\left(\frac{\partial A_{j}}{\partial x_{i}}\right)}=0 .
$$

Equations (4a) and (4b) result in the well-known GL equations. ${ }^{16}$

To find the V-V interaction potential, one has to control the localization and the winding number (also called vorticity) of the vortices. As mentioned earlier, previous theoretical works propose a way to fix the vortices and obtain the interaction potential, based on four steps: (i) fixing a circular phase change of $2 \pi$ around each vortex, (ii) finding the asymptotic behaviors of the vector potential and the amplitude far from each of the vortices ${ }^{1}$ or, equivalently, solving numerically the differential equations for these variables, ${ }^{2}$ (iii) constructing a superposition ansatz for $\Psi$ and $\vec{A}$ that describes the doublevortex structure, and (iv) using the latter ansatz in Eqs. (1) and (3) to obtain the energy for a given separation between vortices.

If we have the asymptotics of the vortices, obtained in step (ii), the integral in Eq. (1) can be solved analytically, giving an analytical expression for the energy as a function of the separation between vortices. In order to find the analytical expression for the asymptotic interaction potential between a vortex and another vortex, an antivortex or a giant vortex, we start from the sequence listed above: A circular phase is fixed around each vortex by assuming $\Psi(r, \theta)=f(r) e^{i n \theta}$, where $(r, \theta)$ are polar coordinates with the origin in the center of the vortex, $n$ is its winding number, and $f(r)$ is the amplitude of its order parameter, which is assumed to be circularly symmetric around the vortex center. Considering the gauge $\vec{A}=n a(r) \widehat{\theta} / r$, the Euler-Lagrange equations (4a) and (4b) for a single vortex read

$$
\frac{d^{2} f}{d r^{2}}+\frac{1}{r} \frac{d f}{d r}-\frac{n^{2}(a-1)^{2}}{r^{2}} f-\frac{\mu^{2}}{2} f\left(f^{2}-1\right)=0
$$

and

$$
\frac{d^{2} a}{d r^{2}}-\frac{1}{r} \frac{d a}{d r}-(a-1) f^{2}=0,
$$

with $f(\infty)=a(\infty)=1$. As is well known, ${ }^{2}$ the winding number $n$ also determines the number of zeros of the vortex field $f(r)$ and, because of the circular symmetry, these zeros must be degenerate at $r=0$. Substituting the auxiliary functions $\sigma(r)=f(r)-1$ and $Q(r)=a(r)-1$ in Eqs. (5a) and (5b), we can suppress the high-order terms in the remaining differential equations when $r \rightarrow \infty$, as $\sigma(\infty)=Q(\infty)=0$, which leads to the following equations, valid in the asymptotic limit:

$$
\left[\frac{d^{2} \sigma}{d(\mu r)^{2}}+\frac{1}{\mu r} \frac{d \sigma}{d(\mu r)}-\sigma\right]=0
$$

and

$$
\frac{d^{2}}{d r^{2}}\left(\frac{Q}{r}\right)+\frac{1}{r} \frac{d}{d r}\left(\frac{Q}{r}\right)-\left(1+\frac{1}{r^{2}}\right)\left(\frac{Q}{r}\right)=0 .
$$

Equations (6a) and (6b) are easily identified as modified Bessel equations and their solutions are $\sigma(r)=\gamma_{1} K_{0}(\mu r)$ and $Q(r)=$ $\gamma_{2} r K_{1}(r)$, where $\gamma_{1}$ and $\gamma_{2}$ are coefficients to be determined. For example, after solving Eqs. (5a) and (5b) numerically, one can obtain these coefficients by fitting $f(r)=1+\gamma_{1} K_{0}(\mu r)$ and $\vec{A}=n\left(1+\gamma_{2} r K_{1}(r)\right) \widehat{\theta} / r$ to the results obtained by the numerical procedure.

Several different procedures can be followed to extract the analytical expression for the interaction potential from these asymptotic functions. As an example, Bettencourt and Rivers ${ }^{9}$ suggested that one can substitute the superposition ansatz $\bar{\Psi}\left(\vec{r}, \overrightarrow{r_{1}}, \overrightarrow{r_{2}}\right)=\Psi\left(\left|\vec{r}-\overrightarrow{r_{1}}\right|\right) \Psi\left(\left|\vec{r}-\overrightarrow{r_{2}}\right|\right)$ and $\overrightarrow{A_{\theta}}\left(\vec{r}, \vec{r}_{1}, \vec{r}_{2}\right)=A_{\theta}\left(\left|\vec{r}-\vec{r}_{1}\right|\right)+A_{\theta}\left(\left|\vec{r}-\overrightarrow{r_{2}}\right|\right)$, for vortices centered at $\vec{r}_{1}$ and $\overrightarrow{r_{2}}$, in the energy functional in Eq. (1), and keep only the terms that are linear in the fields for each vortex. After some calculations described in more detail in Ref. 9, one obtains

$$
E_{\text {int }}(d)=2\left[n_{1} n_{2} \gamma_{2}^{(1)} \gamma_{2}^{(2)} K_{0}(d)-\gamma_{1}^{(1)} \gamma_{1}^{(2)} K_{0}(\mu d)\right],
$$

where $d$ is the separation between vortices, $n_{i}$ is the winding number, and $\gamma_{1}^{(i)}$ and $\gamma_{2}^{(i)}$ are the fitting coefficients for the vortex $i$ in position $\vec{r}_{i}$. We point out that in the paper by Bettencourt and Rivers, the expression for $E_{\text {int }}$ is slightly different from Eq. (7) because they assumed that $\gamma_{1}^{(i)}=$ $\left|n_{i}\right| \gamma_{2}^{(i)}$, which is valid only in the critical coupling regime for $\mu=1$, as stressed by Speight ${ }^{7}$ and verified by Bogomol'nyi equations for this regime. The same expression for $E_{\text {int }}(d)$ was found by Kramer by a perturbational approach ${ }^{1}$ and can also be obtained by considering point sources in a linearized field theory. ${ }^{7,8}$

It can be easily seen that the potential in Eq. (7) is consistent with the fact that for $\mu<1(>1)$, the $\mathrm{V}-\mathrm{V}$ interaction is 
attractive (repulsive) for large separation, leading to a type-I (type-II) superconducting behavior. This statement is also valid for V-GV interactions, as Eq. (7) still holds for this case, where the interaction potential is obtained just by setting $n_{1}=1$ and $n_{2}>1$ and finding the fitting coefficients $\gamma_{1}^{(i)}$ and $\gamma_{2}^{(i)}$ for this case. However, for a V-AV interaction, $n_{1} n_{2}$ is always negative, leading to an attractive potential $E_{\text {int }}(d)$ for any value of $\kappa$. Furthermore, Eq. (7) shows that the interaction between an antivortex and giant vortex is always attractive as well. This can be understood through a heuristic argument: When a vortex and an antivortex are far from each other, the energy of the system is nonzero, as it is the sum of the energies of one vortex and one antivortex; on the other hand, when they approach each other they should annihilate, giving zero energy. Hence, at least at some distance, the energy of the $\mathrm{V}$-AV pair must decrease as $d$ approaches zero and, as a result, the interaction potential is attractive. Indeed, evidences of $\mathrm{V}-\mathrm{AV}$ attraction for any value of $\kappa$ were already observed in previous studies on $\mathrm{V}$-AV scattering in bulk superconductors. ${ }^{17,18}$ However, this result is in contradiction with the conjecture proposed by Ref. 19, which claims that in type-I superconductors the interaction of a V-AV pair is repulsive, and which was used to explain the stability a V-AV molecule in mesoscopic superconducting triangles. ${ }^{20,21}$ As follows from our theory, the V-AV interaction is always attractive and should lead to a disfavored $\mathrm{V}-\mathrm{AV}$ molecule in type-I superconducting polygons, because in that case the $\mathrm{V}-\mathrm{V}$ interaction is also attractive. ${ }^{22,23}$

One more question arises from the conjecture of Ref. 19: If vortices attract (repel) each other in type-I (type-II) superconductors, whereas exactly the opposite occurs for V-AV interactions, what one would expect in a critically coupled system? In this regime, i.e., when $\mu=1$, vortices do not interact; should we expect the same for V-AV? The answer to this question is provided by the Bogomol'nyi equations ${ }^{24}$

$$
\begin{gathered}
{\left[\left(\frac{\partial}{\partial x_{1}}-i A_{1}\right)+\operatorname{sgn}(n) i\left(\frac{\partial}{\partial x_{2}}-i A_{2}\right)\right] \Psi=0,} \\
|\vec{\nabla} \times \vec{A}|+\operatorname{sgn}(n) \frac{1}{2}\left(|\Psi|^{2}-1\right)=0 .
\end{gathered}
$$

For the single vortex ansatz chosen before, $\Psi(r, \theta)=f(r) e^{i n \theta}$ and $\vec{A}=n a(r) \widehat{\theta} / r$, these equations read ${ }^{25}$

$$
\begin{aligned}
& r \frac{d f}{d r}-\operatorname{sgn}(n) n(1-a) f=0, \\
& \frac{2 n}{r} \frac{d a}{d r}+\operatorname{sgn}(n)\left(f^{2}-1\right)=0 .
\end{aligned}
$$

Substituting the latter formulas in Eq. (9) and neglecting higher-order terms in $\sigma(r)$ and $Q(r)$ yields $n Q=-\operatorname{sgn}(n) r \frac{d \sigma}{d r}$ or, using the asymptotic forms of these functions, $n \gamma_{2} r K_{1}(r)=-\operatorname{sgn}(n) \gamma_{1} r d K_{0}(r) / d r=\operatorname{sgn}(n) \gamma_{1} r K_{1}(r) \Rightarrow$ $\gamma_{1}=\operatorname{sgn}(n) n \gamma_{2}$. Substituting this expression for $\gamma_{1}^{(i)}$ in Eq. (7) shows that the interaction potential for $\mathrm{V}-\mathrm{AV}$ in the critical coupling regime is $E_{\text {int }}(d)=4 n_{1} \gamma_{2}^{(1)} n_{2} \gamma_{2}^{(2)} K_{0}(d)$, which is still attractive because $n_{1} n_{2}<0$. Hence, unlike vortex-vortex pairs, a V-AV pair exhibits an attractive interaction even in the critical case of $\mu=1$.

Notice that the V-AV interaction potential in our work is derived for a bulk superconductor, in the absence of an external magnetic field. On the other hand, the V-AV molecules shown in Refs. 19-21 were found in a different situation, namely, in mesoscopic samples under applied external fields, so that the net magnetic field in the region of the antivortex was actually positive. Besides, in mesoscopic samples as those of Refs. 1921, an effective repulsion of vortices and antivortices could result from the interaction with their images for certain (usually unstable) configurations. Therefore, our results call in question just the general statement and motivation of Refs. 19-21 that a vortex and an antivortex repel in type-I superconductors, because we have shown that, at least in bulk samples, this is not the case. Other reasons, related to the quantum confinement and the resulting electromagnetic fields, should be exploited for the explanation of the increased stability of V-AV molecules found in Refs. 19-21, which makes their findings even more intriguing.

It should be mentioned that, as $\gamma_{1} \neq \gamma_{2}$ for $\mu \neq 1$, the interaction potential given by Eq. (7) may diverge at small distances for some values of $\mu$. This signals the breakdown of this analytical expression for small $d$. Actually, for small separation $d$, the superposition ansatz proposed by Bettencourt and Rivers and used in the present section also fails, because it does not take into account either the spatial deformation of the fields, or the possibility of the formation of giant vortices. $^{2}$ Hence, the analytical expression for the interaction potential between vortices has a validity restricted to large $d$. Nevertheless, when the interaction potential is calculated by numerical means, taking into account all the features mentioned above, the result shows very good agreement with Eq. (7) for $d$ larger than a critical separation $d_{c}$ that depends on $\mu$, as will be shown in the next section.

The interaction force $\Omega$ can be obtained by taking the derivative of the energies with respect to the distance between the two vortices. The results for $\mu=1$ in the V-V and V-GV cases clearly give $\Omega(d)=0$. The analytical expression for the force for large vortex-vortex separation can be easily derived from Eq. (7) as

$$
\Omega(d)=2\left[n_{1} n_{2} \gamma_{2}^{(1)} \gamma_{2}^{(2)} K_{1}(d)-\gamma_{1}^{(1)} \gamma_{1}^{(2)} \mu K_{1}(\mu d)\right] .
$$

\section{GL EQUATIONS FOR FIXED VORTICES}

We recall step (i) in the procedure described in the previous section for obtaining the $\mathrm{V}-\mathrm{V}$ interaction, which is fixing a circular phase change around each vortex. In the present section, we derive the Euler-Lagrange equations from Eqs. (4a) and (4b) with the constraints imposed by step (i).

In the paper by Jacobs and Rebbi, ${ }^{2}$ the authors fixed the phase for a single vortex and obtained "modified" GL equations, given by Eq. (2.18) of their paper or, equivalently, Eqs. (5a) and (5b) of the present work, as well as Eq. (7) of the paper by Babaev and Speight. ${ }^{14}$ Although they presented these equations in their paper, Jacobs and Rebbi did not solve them directly, but used instead variational functions for $f(r)$ and $a(r)$ and minimized the energy $E$ without solving the differential equations.

For the two-vortex system, Jacobs and Rebbi made a different ansatz, $\Psi=\exp \left[i \theta_{1}\right] \exp \left[i \theta_{2}\right] f(r, \theta)$, where $\theta_{1}$ and $\theta_{2}$ are azimuthal angles around each vortex position, $f$ is a real function that is not necessarily circularly symmetric and is zero at the position of each vortex, and the winding numbers were chosen as 1 for each vortex. Having fixed the positions 
and vorticities, they just needed to find $f$ and $\vec{A}$ that minimize $E$. As before, instead of deriving Euler-Lagrange (differential) equations, they used a variational procedure, considering trial functions that account for the deformation of the vortices toward the formation of the giant vortex.

The results of Jacobs and Rebbi are rather accurate and the advantage of their approach is that many terms of the variational function can be integrated analytically. However, the variational procedure involves many parameters, it is a very long analytical calculation, and their trial function is not the most general function: If one wants to solve the problem for a V-AV or a V-GV pair, the trial function has to be modified and consequently also the analytical integrals in the variational procedure.

In order to obtain the intervortex potential, our approach starts from the ansatz for two vortices $\Psi=e^{i n_{1} \theta_{1}} e^{i n_{2} \theta_{2}} f(x, y)$, where we control the vorticity $n_{1}$ and $n_{2}$ of each vortex. We further rewrite $e^{i n_{j} \theta_{j}}$ in Cartesian coordinates:

$$
e^{i n_{j} \theta_{j}}=\left(\frac{x_{j}+i y_{j}}{x_{j}-i y_{j}}\right)^{n_{j} / 2}
$$

where $\vec{r}_{j}=\left(x_{j}, y_{j}, 0\right)$ is the in-plane position vector with origin in the center of the vortex $j$. As we will study the case for two vortices separated by a distance $d$, we take $\vec{r}_{1}=(x-d / 2, y, 0)$ and $\vec{r}_{2}=(x+d / 2, y, 0)$.

Next, we substitute this ansatz into Eq. (3) to get the energy functional $F$ for fixed position of vortices as

$$
\begin{aligned}
F= & \frac{1}{2}\left[\left(\frac{\partial f}{\partial x}\right)^{2}+\left(\frac{\partial f}{\partial y}\right)^{2}\right] \\
& +\frac{1}{2} f^{2}\left[\bar{X}^{2}+\bar{Y}^{2}+2\left(A_{x} \bar{Y}-A_{y} \bar{X}\right)+A^{2}\right] \\
& +\frac{\mu^{2}}{8}\left(1-f^{2}\right)^{2}+\frac{1}{2}|\vec{\nabla} \times \vec{A}|^{2},
\end{aligned}
$$

where

$$
\bar{X}=\frac{n_{1} x_{1}}{r_{1}^{2}}+\frac{n_{2} x_{2}}{r_{2}^{2}}, \quad \bar{Y}=\frac{n_{1} y_{1}}{r_{1}^{2}}+\frac{n_{2} y_{2}}{r_{2}^{2}} .
$$

Notice that although $\bar{X}$ and $\bar{Y}$ seem to have no physical meaning, they can be related to the gauge proposed by Jacobs and Rebbi for the vector potential, $\vec{A}=n a(r) \widehat{\theta} / r$, or $\vec{A}=(-n \sin \theta / r, n \cos \theta / r, 0)=\left(-n y / r^{2}, n x / r^{2}, 0\right)$ in Cartesian coordinates, which was shown to be compatible with the symmetry of the Euler-Lagrange equations, leading to Eqs. (5a) and (5b) for a single vortex. However, as we are looking for general differential equations for two vortices, we will not make any a priori choice of gauge for the vector potential.

In previous works, ${ }^{2,14}$ the Euler-Lagrange equations were not explicitly derived. We derived the Euler-Lagrange equations for the present problem setting, which are given by

$$
\begin{gathered}
\nabla^{2} f-\left[\bar{X}^{2}+\bar{Y}^{2}+2\left(A_{x} \bar{Y}-A_{y} \bar{X}\right)+A^{2}\right] f \\
+\frac{\mu^{2}}{2}\left(1-f^{2}\right) f=0
\end{gathered}
$$

and

$$
\vec{\nabla} \times \vec{\nabla} \times \vec{A}+\left[\vec{A}-\frac{n_{1} \widehat{\theta_{1}}}{r_{1}}-\frac{n_{2} \widehat{\theta_{2}}}{r_{2}}\right] f^{2}=0,
$$

where the unitary angular vectors around each vortex can be rewritten as $\widehat{\theta_{j}}=\left(-y_{j} / r_{j}, x_{j} / r_{j}, 0\right)$. One can even verify, after some manipulations of the equations, that inserting $n_{2}=0$ in these equations leads to Eq. (2.18) in the paper by Jacobs and Rebbi, which is the equation for a single vortex, but in Cartesian coordinates. Solving Eqs. (13a) and (13b) is as equally demanding as solving the common GL equations, which was done in many works in the literature. . $^{3,22,23}$

\section{NUMERICAL RESULTS AND FITTING FUNCTIONS}

We solved Eqs. (13a) and (13b) numerically using the finite-difference technique and a relaxation method suitable for nonlinear differential equations. ${ }^{26}$ The two-dimensional system is divided in a uniform square $601 \times 601$ grid with total dimensions $60 \lambda \times 60 \lambda$. The singularities in the amplitude of the order parameter appear naturally in the center of each vortex position, as a consequence of the fixed circular phase $e^{i n_{i} \theta_{i}}$ defined around each vortex $i$, which guarantees the existence of zeros of the order parameter in the center of the vortices. $^{2}$ The results for $\mathrm{V}-\mathrm{V}, \mathrm{V}-\mathrm{GV}$, and $\mathrm{V}$-AV interactions are presented separately in the following subsections. Analytical fitting functions will be proposed for the numerically obtained curves, where the fitting error is defined by the variance ${ }^{26}$

$$
v=\sum_{n=1}^{N} \frac{\left[G(n)-G_{\mathrm{fit}}(n)\right]^{2}}{\left(N-N_{p}\right)},
$$

where $G(n)$ is the numerical data set, $G_{\text {fit }}(n)$ is the analytical fitting function, $N$ is the length of the data set, and $N_{p}$ is the number of variational parameters of the fitting function. ${ }^{27}$

\section{A. Vortex-vortex interaction}

The numerical results for the $\mathrm{V}-\mathrm{V}$ interaction force are shown in Fig. 1 for several values of $\mu$ in the type-II (a) and type-I (b) regimes. Notice that for $\mu \approx 0$ vortices should not interact and the force vanishes, but for $0<\mu<1$ they attract (type-I regime) and the force is negative in this case. However, at the critical point $\mu=1$ the force vanishes again. Hence, in the type-I case, two different regimes can be identified: one where the force increases from zero, as $\mu$ increases from zero, and the other when the force decreases back to zero, as $\mu$ approaches 1 . This can be seen from Fig. 1(c), where the extremum of the force peak $\Omega_{\max }$ (open triangles, left-hand scale) increases with $\mu$ for small $\mu$, but for $0.6<\mu<1$ the peak decreases with $\mu$, approaching zero when $\mu=1$. The numerical results can be fitted to $\Omega_{\max }(\mu)=0.0961 \mu(\mu-1) /(1+0.2863 \mu)^{1.341}$, which is shown by the red curve in Fig. 1(c). From Figs. 1(a) and $1(\mathrm{~b})$ we see that the force exhibits a maximum at some critical separation $d_{c}$, which depends on the GL parameter $\mu=\sqrt{2} \kappa$. The critical separation $d_{c}$ is also shown in Fig. 1(c) (open squares, right-hand scale) as a function of $\mu$. The fit of this curve, given by $d_{c}=22.203(1+10.504 \mu)^{-0.774}$ (with an estimated variance $v \approx 0.3 \%$ ), is shown by the solid curve in Fig. 1(c), which suggests that the critical separation for the $\mathrm{V}-\mathrm{V}$ interaction approaches zero in the extreme type-II situation $(\mu \rightarrow \infty)$. An effective extreme type-II scenario can also be achieved in a superconducting film of thickness $w \ll \lambda$, where the effective penetration depth is $\Lambda=\lambda^{2} / w$, and for 

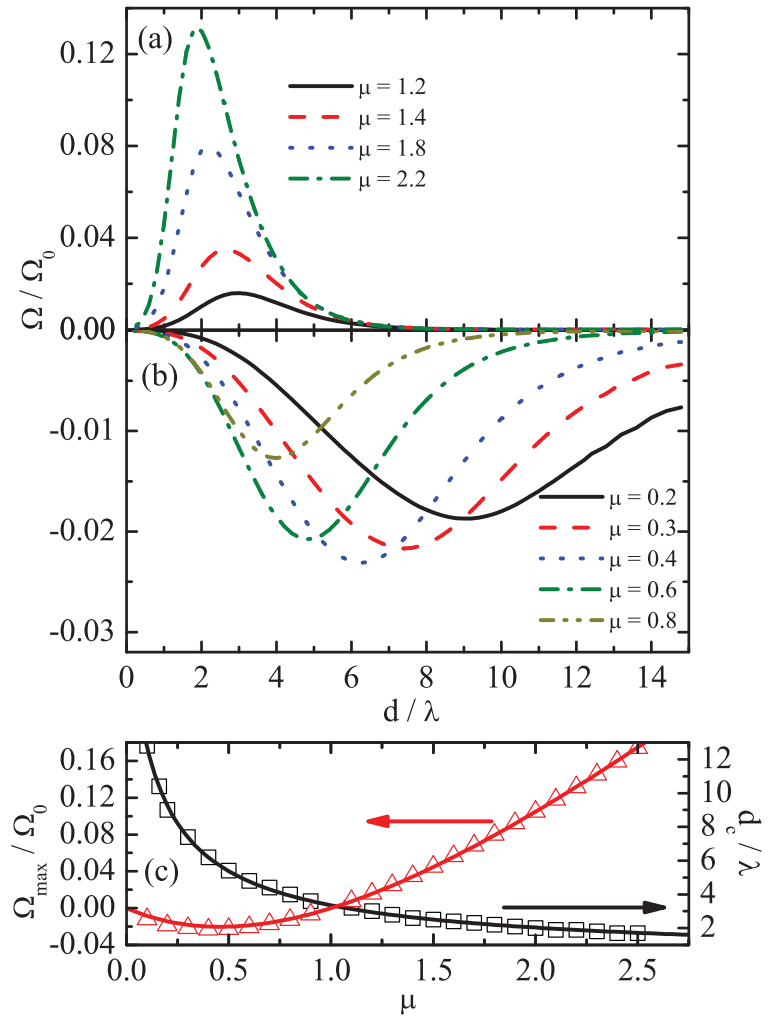

FIG. 1. (Color online) Numerically obtained V-V interaction force $\Omega$ as a function of the separation $d$ between vortices, for several values of $\mu=\sqrt{2} \kappa$ in the (a) type-II and (b) type-I regimes. (c) Critical separation $d_{c}$ (open squares, right-hand scale) and extremum $\Omega_{\max }$ (open triangles, left-hand scale), which correspond respectively to the position and amplitude of the peak in the force, as a function of the GL parameter. The fitting functions for $d_{c}$ and $\Omega_{\max }$ are plotted by the solid curves.

which analytical expressions for the V-V interaction force were proposed by Pearl ${ }^{28}$ and later by Brandt. ${ }^{29}$ However, in the case of superconducting films, the $\mathrm{V}-\mathrm{V}$ force decays monotonically as $1 / d^{2}$, while in the present case of a bulk superconductor the decay is exponential. Hence, although both situations can be considered as extreme type-II limits, our results for bulk superconductors with $\mu \rightarrow \infty$ are quantitatively different from those for thin superconducting films.

The importance of solving Eqs. (13a) and (13b) numerically for two separate vortices lies in the possibility of obtaining the interaction force between vortices even in the small separation limit, which cannot be described by the asymptotic functions given in the literature ${ }^{9}$ and described by Eq. (10). However, because solving these equations is generally not an easy task, we attempt to propose here an analytical expression that possesses all the features of the numerically obtained force as a function of the vortex separation. Such an analytical expression can be helpful, e.g., for numerical modeling of vortex structures by means of MD simulations, where vortices are considered as point particles. Up to now, in those MD simulations one typically uses the asymptotic analytical expressions for the $\mathrm{V}-\mathrm{V}$ interaction that are only valid in the large separation limit. ${ }^{30,31}$ To obtain a proper fitting function, we first analyze separately the behaviors for large and small vortex separations. For large separation, using the asymptotic form of the modified Bessel functions, Eq. (10) can be rewritten as

$$
\Omega(d \rightarrow \infty)=\gamma d^{-1 / 2}\left(\delta e^{-d}-\sqrt{\mu} e^{-\mu d}\right),
$$

where $\gamma$ and $\delta$ are fitting parameters. For small separation, our results show that a power function of $d$ describes the force satisfactorily, i.e.,

$$
\Omega(d \rightarrow 0)=p d^{q},
$$

with $p$ and $q$ as fitting parameters. Two examples of such fittings are shown in Fig. 2 for $\mu=0.6$ (bottom) and $\mu=1.7$ (top). Notice that the parameter $q$ depends weakly on $\mu$, exhibiting values between $\approx 2.7$ and $\approx 2.8$ for all values of $\mu$ considered in the $\mathrm{V}-\mathrm{V}$ case.

Following the established behavior of the interaction in the limiting cases, we propose a single function that has the above limits as limiting behaviors:

$$
\Omega_{\mathrm{fit}}(d)=\eta_{1} \frac{d^{\eta_{3}}}{1+\eta_{2} d^{\eta_{3}+(1 / 2)}}\left(\eta_{4} e^{-d}-\sqrt{\mu} e^{-\mu d}\right),
$$

where $\eta_{i}(i=1-4)$ are four fitting parameters.

Figure 3 shows the fitting obtained with Eq. (17) for the same values of $\mu$ presented in Fig. 2. The fitting is not ideal for $d<\lambda$, where the force becomes very small. Nevertheless, we found that the estimated variance, as calculated by Eq. (14),

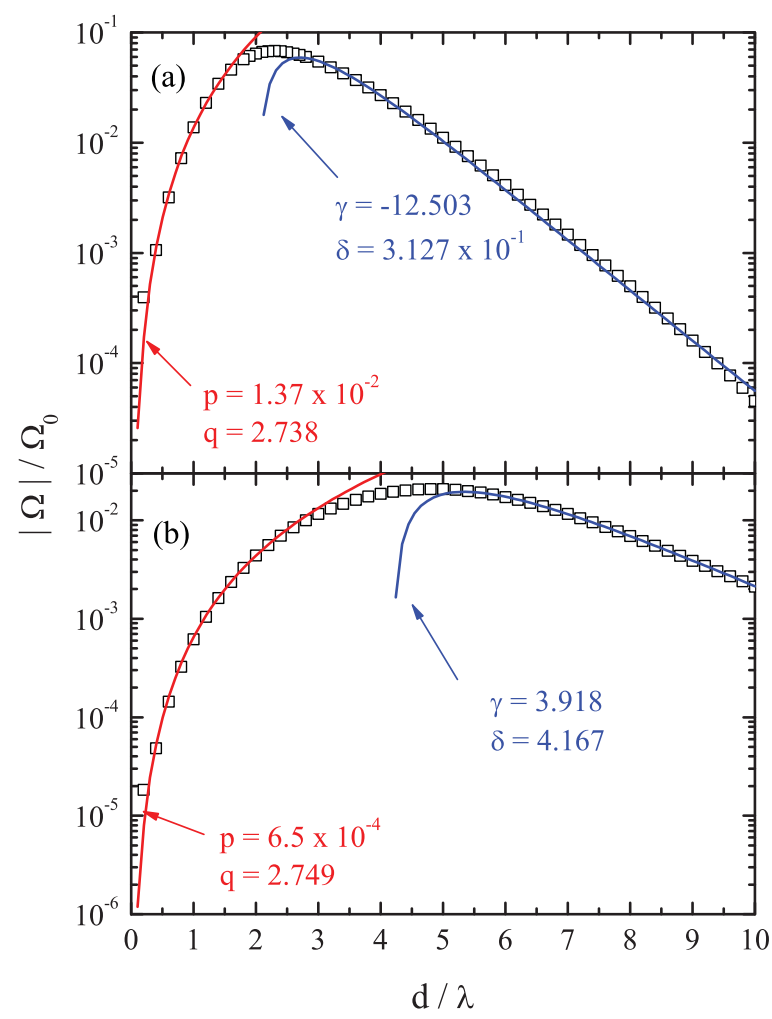

FIG. 2. (Color online) Comparison between the V-V interaction force as a function of the separation $d$ obtained by the numerical method (symbols) and by the asymptotic expressions (curves) from Eqs. (15) and (16), for (a) $\mu=1.7$ and (b) $\mu=0.6$. The forces are plotted on a $\log _{10}$ scale and the values of the fitting parameters $p, q, \gamma$, and $\delta$ are given in each panel. 


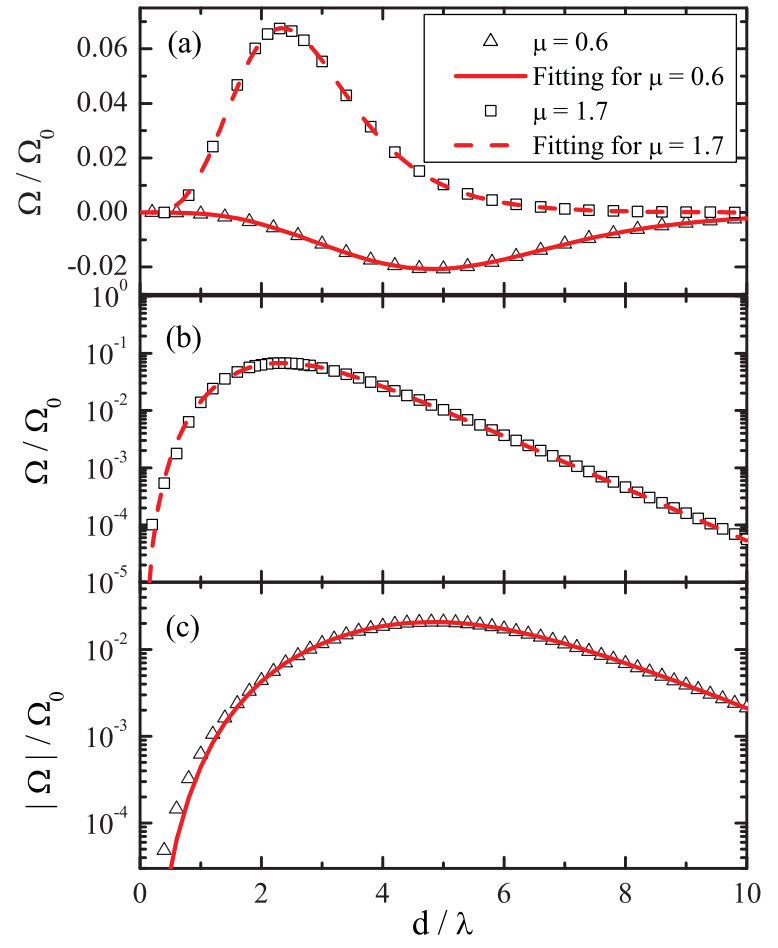

FIG. 3. (Color online) (a) Comparison between the V-V interaction force as a function of the separation $d$ obtained by the numerical method (symbols) and by the fitting function (curves) given by Eq. (17). The solid (dashed) curves and the open triangles (squares) are the results for $\mu=0.6$ (1.7). (b) and (c) The results for each $\mu$ are plotted separately in a log scale to emphasize the maximal deviation of the fitting function from the data.

is $\sim 10^{-9}\left(10^{-7}\right)$ for $\mu=0.6(\mu=1.7)$. In fact, although the numerical and fitting curves differ by a factor of $2-3$ for $d<\lambda$, the interaction force in this region is extremely small, so this discrepancy does not influence the overall estimated variance. Please note that the V-V interaction potential, which is an integrated force, will be even more accurate.

The values of the four fitting parameters are given in Table I for $\mu$ from 0.2 to 2.5. Notice that the estimated variance $v$ increases with $\mu$, and thus Eq. (17) should not be used in the extreme type-II case. Nevertheless, in the extreme type-II case the critical separation $d_{c}$ approaches zero, as mentioned earlier, and consequently, the short-range part of the V-V interaction force will not be important in such a situation. Hence, the asymptotic expression $\Omega(d)=f_{0} K_{1}(d)$ frequently used in the literature, ${ }^{32,33}$ which can be obtained from Eq. (10) by making $\mu \rightarrow \infty$, is expected to provide a good description of the V-V interaction force in extreme type-II situations.

We next attempt to find an analytical expression for the fitting parameters as a function of $\mu$. Their dependence on $\mu$ is shown in Fig. 4. Three different ranges of $\mu$, delimited by vertical dotted lines in Fig. 4, can be distinguished. The physical reason for the existence of three different behaviors of the parameters $\eta_{i}$ as a function of $\mu$ is the following: For type-I $(\mu<1)$, as we explained earlier, there must be a regime where the size of the attractive force peak increases with $\mu$ and another region where it decreases with $\mu$. This defines the ranges $1(\mu<0.5)$ and $2(0.5<\mu<1)$, respectively. Range 3
TABLE I. Fitting parameters $\eta_{i}$ and the estimated variance $v$ for Eq. (17) in the V-V case for different values of $\mu$.

\begin{tabular}{lllcrc}
\hline \hline$\mu$ & \multicolumn{1}{c}{$\eta_{1}$} & \multicolumn{1}{c}{$\eta_{2}$} & $\eta_{3}$ & $\eta_{4}$ & $v\left(\times 10^{-8}\right)$ \\
\hline 0.2 & $6.564 \times 10^{-7}$ & $5.13 \times 10^{-7}$ & 6.135 & -83.611 & 1.42 \\
0.3 & $1.522 \times 10^{-5}$ & $7.268 \times 10^{-6}$ & 5.213 & -17.667 & 3.96 \\
0.4 & $5.698 \times 10^{-5}$ & $2.041 \times 10^{-5}$ & 4.950 & -8.014 & 5.06 \\
0.5 & $1.284 \times 10^{-4}$ & $3.911 \times 10^{-5}$ & 4.796 & -5.090 & 1.95 \\
0.6 & $4.474 \times 10^{-4}$ & $1.222 \times 10^{-4}$ & 4.440 & -1.538 & 0.448 \\
0.7 & $1.62 \times 10^{-3}$ & $3.968 \times 10^{-4}$ & 4.046 & 0.237 & 0.239 \\
0.8 & $4.12 \times 10^{-3}$ & $9.326 \times 10^{-4}$ & 3.760 & 0.766 & 0.215 \\
0.9 & $8.46 \times 10^{-3}$ & $1.79 \times 10^{-3}$ & 3.544 & 0.943 & 0.116 \\
1.1 & $1.546 \times 10^{-2}$ & $3.88 \times 10^{-3}$ & 3.489 & 1.049 & 0.139 \\
1.2 & $2.068 \times 10^{-2}$ & $5.37 \times 10^{-3}$ & 3.443 & 1.095 & 0.98 \\
1.3 & $2.67 \times 10^{-2}$ & $7.31 \times 10^{-3}$ & 3.410 & 1.140 & 3.16 \\
1.4 & $3.369 \times 10^{-2}$ & $9.78 \times 10^{-3}$ & 3.382 & 1.183 & 7.24 \\
1.5 & $4.175 \times 10^{-2}$ & $1.286 \times 10^{-2}$ & 3.358 & 1.225 & 13.8 \\
1.6 & $5.094 \times 10^{-2}$ & $1.664 \times 10^{-2}$ & 3.338 & 1.265 & 23.6 \\
1.7 & $6.136 \times 10^{-2}$ & $2.121 \times 10^{-2}$ & 3.320 & 1.304 & 36.8 \\
1.8 & $7.308 \times 10^{-2}$ & $2.667 \times 10^{-2}$ & 3.306 & 1.342 & 53.9 \\
1.9 & $8.618 \times 10^{-2}$ & $3.311 \times 10^{-2}$ & 3.294 & 1.378 & 75.3 \\
2.0 & 0.1008 & $4.066 \times 10^{-2}$ & 3.283 & 1.414 & 101.1 \\
2.1 & 0.1169 & $4.94 \times 10^{-2}$ & 3.275 & 1.449 & 131.6 \\
2.2 & 0.1347 & $5.945 \times 10^{-2}$ & 3.268 & 1.483 & 166.7 \\
2.3 & 0.1542 & $7.093 \times 10^{-2}$ & 3.262 & 1.517 & 206.6 \\
2.4 & 0.1756 & $8.396 \times 10^{-2}$ & 3.258 & 1.549 & 251.1 \\
2.5 & 0.199 & $9.866 \times 10^{-2}$ & 3.254 & 1.581 & 286.2 \\
\hline \hline
\end{tabular}

is then the type-II regime, for $\mu>1$, where the interaction force is repulsive. The functions $\eta_{i}(\mu)$ in Fig. 4 were fitted as

and

$$
\begin{gathered}
\eta_{1}(\mu)=e^{B_{1}\left(\mu^{C_{1}}+A_{1}\right)}, \\
\eta_{2}(\mu)=e^{B_{2}\left(\mu^{C_{2}}+A_{2}\right)}, \\
\eta_{3}(\mu)=A_{3}+B_{3} e^{C_{3} \mu},
\end{gathered}
$$

$$
\eta_{4}(\mu)=A_{4}+B_{4} \mu^{C_{4}}
$$

with different parameters $A_{i}, B_{i}$, and $C_{i}$ for each range listed in Table II. These fitting functions for $\eta_{i}(\mu)$ are shown as solid curves in Fig. 4. Notice that the parameter $\eta_{4}$ must satisfy the condition $\eta_{4} \leqslant \sqrt{\mu}(\geqslant \sqrt{\mu})$ in the type-I (type-II) case, otherwise the difference between the exponential terms in Eq. (17) would exhibit a sign change for small separations, leading to a spurious repulsive (attractive) region in this case. In the type-II case, this condition leads to $\eta_{4}(\mu) \approx \sqrt{\mu}$ as the best value for this fitting parameter.

It is important to point out that the results obtained for $\eta_{2}$ are not the same as the values of $q$ in Eq. (16) for the power law at small separations, which, as mentioned earlier, are between $\approx 2.7$ and $\approx 2.8$. This is reasonable, because the exponential terms in Eq. (17) still play a role in the small $d$ limit of this expression, thus the parameter $\eta_{2}$ must assume a value that is different from $q$ in order to compensate these terms. The values obtained for $\eta_{4}$, which is the parameter controlling the large $d$ range of Eq. (10), are also not the same as the values obtained when one uses the asymptotics of each single vortex to find the parameters $\gamma_{1}^{(i)}$ and $\gamma_{2}^{(i)}$ in Eq. (10). Actually, for $\mu>1$, we found $\eta_{4} \approx \sqrt{\mu}$, which is equivalent to making $\gamma_{1}^{(i)}=\gamma_{2}^{(i)}$. As 


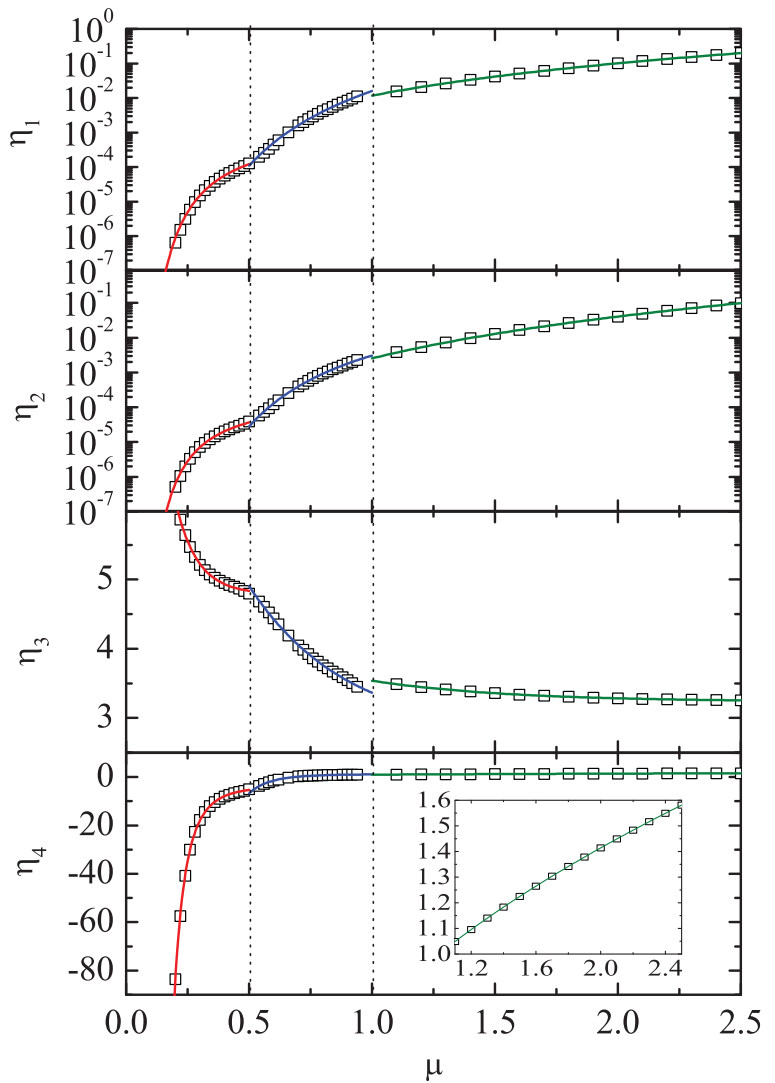

FIG. 4. (Color online) Fitting parameters (symbols) in Eq. (17) as a function of $\mu=\sqrt{2} \kappa$ for the $\mathrm{V}-\mathrm{V}$ case. The curves are the fitting functions given by Eqs. (18) for $\eta_{i}(\mu)(i=1-4)$ in three different regions: $0<\mu<0.5,0.5<\mu<1$, and $1<\mu$. The inset shows a magnification of the results for $\eta_{4}$ at large $\mu$.

$\mu$ increases, the difference between $\gamma_{1}^{(i)}$ and $\gamma_{2}^{(i)}$ is amplified, ${ }^{7}$ leading to a higher variance $v$ for large $\mu$, as shown in Table I. Even so, this choice of $\eta_{4}$ conveniently leads to a function that decays exponentially for large separation $d$, as expected for $\mathrm{V}-\mathrm{V}$ interactions in bulk superconductors, and which exhibits no sign change at small separations. Of course, the fitting function Eq. (17) can be improved to provide a better fitting of the large separation part and to reproduce a perfect power

TABLE II. Fitting parameters in Eqs. (18a)-(18d) for the V-V case for three different ranges of $\mu$.

\begin{tabular}{lclc}
\hline \hline Parameter & $\mu<0.5$ & $0.5<\mu<1$ & $\mu>1$ \\
\hline$A_{1}$ & 5.977 & -0.5420 & -0.9404 \\
$B_{1}$ & -1.092 & -9.041 & -74.584 \\
$C_{1}$ & -1.191 & -0.6323 & $-4.221 \times 10^{-2}$ \\
$A_{2}$ & 13.845 & $7.935 \times 10^{-2}$ & -0.9843 \\
$B_{2}$ & -0.6218 & -5.359 & -379.321 \\
$C_{2}$ & -1.373 & -0.9084 & $-1.057 \times 10^{-2}$ \\
$A_{3}$ & 4.79 & 2.756 & 3.234 \\
$B_{3}$ & 12.542 & 7.587 & 1.849 \\
$C_{3}$ & -11.183 & -2.523 & -1.804 \\
$A_{4}$ & -3.677 & 1.215 & 0 \\
$B_{4}$ & $-8.663 \times 10^{-2}$ & -0.1229 & 1 \\
$C_{4}$ & -4.244 & -6.022 & 0.5 \\
\hline \hline
\end{tabular}

law for small separations, but this would require more fitting parameters and very complicated expressions. Equation (17) is simple and still accurate for $0 \leqslant \mu \leqslant 2.5$, as verified by the small variances $v<10^{-6}$ in Table I and by the comparison with the numerical results in Fig. 3.

\section{B. Vortex-giant vortex interaction}

As observed in Fig. 1, the interaction force between two vortices shows a maximum at some critical separation $d_{c}$ and decays to zero for both very large and very small separations. The former is reasonable, because the interaction between vortices is expected to weaken as they are placed further from each other. The latter is owing to the formation of a giant vortex state: When two vortices of winding numbers, e.g., $n_{1}=1$ and $n_{2}=1$ are put close to each other, they coalesce, forming a $n=n_{1}+n_{2}=2$ giant vortex. ${ }^{2-5}$ In the absence of lateral confinement, a giant vortex is a stable (unstable) state in type-I (type-II) systems and can interact as such with other vortices, and this motivated us to investigate the interaction force between a vortex and a giant vortex.

The V-GV interaction force is shown in Fig. 5 as a function of the distance between them, for several values of $\mu$ in the type-II (a) and type-I (b) regimes. The behavior of the curves is quite similar to those in Fig. 1 for the V-V case, but with different amplitudes and critical separations. The critical
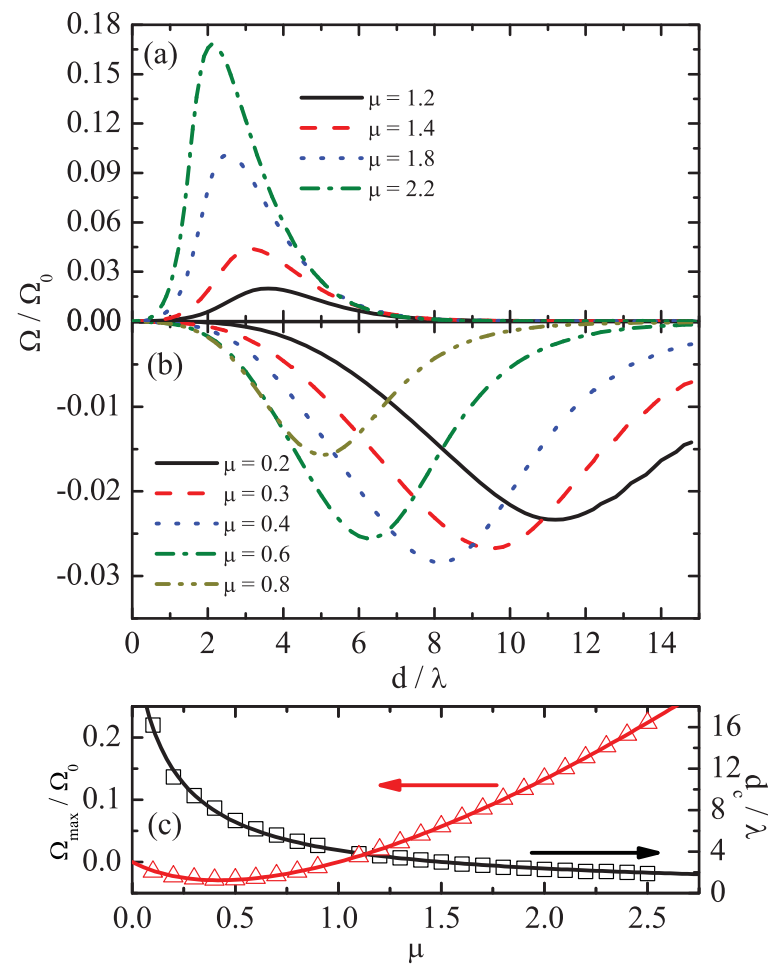

FIG. 5. (Color online) Numerically obtained V-GV interaction force $\Omega$ as a function of the separation $d$ between a vortex and a double-quantized vortex for several values of $\mu=\sqrt{2} \kappa$ in the (a) type-II and (b) type-I regimes. (c) Critical separation $d_{c}$ (open squares, right-hand scale) and extremum $\Omega_{\max }$ (open triangles, left-hand scale), which correspond respectively to the position and amplitude of the peak in the force, as a function of the GL parameter. The fitting functions for $d_{c}$ and $\Omega_{\max }$ are plotted by the solid curves. 
separation $d_{c}$, beyond which the vortices start to coalesce, obtained numerically for the $\mathrm{V}-\mathrm{GV}$ interaction, is shown as a function of the GL parameter $\mu$ in Fig. 5(c), along with its fitting function $d_{c}=25.043(1+6.632 \mu)^{-0.8862}$ (with an estimated variance $v \approx 2 \%$ ). Notice that the critical separation for the $\mathrm{V}-\mathrm{GV}$ interaction is always larger than the one for the $\mathrm{V}-\mathrm{V}$ case, because the giant vortex has a larger core in comparison to a $n=1$ vortex. Nevertheless, the fitting function shows that the smallest critical separation for the $\mathrm{V}-\mathrm{GV}$ interaction force, which would be obtained in an extreme type-II regime, is also $d_{c}(\mu \rightarrow \infty)=0$, as in the $\mathrm{V}-\mathrm{V}$ case. The behavior of the extremum of the force peak $\Omega_{\max }$ as a function of $\mu$, shown as open triangles in Fig. 5(c), is similar to that found for the $\mathrm{V}-\mathrm{V}$ case, with the amplitude approaching zero for $\mu \rightarrow 0$ and $\mu \rightarrow 1$, and increasing monotonically for $\mu$ increasing above 1 . The extremum of the force peak can be fitted to $\Omega_{\max }=0.1709 \mu(\mu-1) /(1+1.854 \mu)^{0.6087}$, which is shown by the solid curve in Fig. 5(c).

In Sec. II, we analytically found that Eq. (10) remains valid for the asymptotic V-GV interactions, simply by choosing $n_{1}=1, n_{2}=2$ and changing the parameters $\gamma_{1}^{(i)}$ and $\gamma_{2}^{(i)}$ accordingly. Moreover, our results show that the force in the small separation limit in this case can still be well described by a power function of the separation $d$. The fitting of the force for the small and large separation limits, given by Eqs. (15) and (16), are shown in Fig. 6 for the V-GV interaction, agreeing

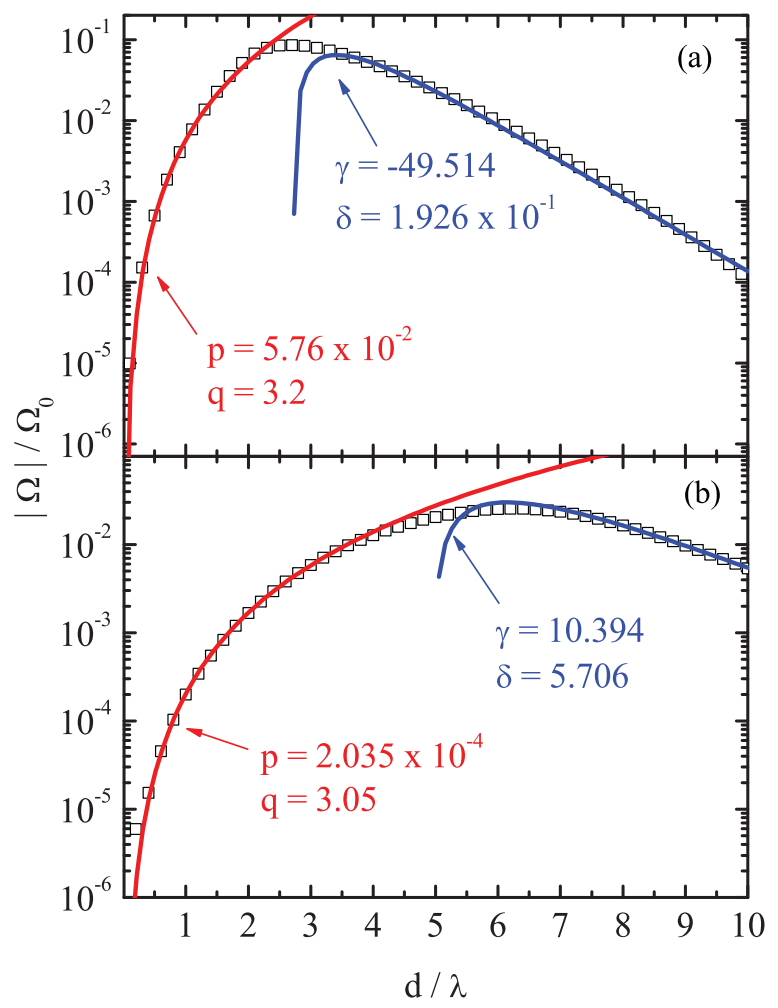

FIG. 6. (Color online) Comparison between the V-GV interaction force as a function of the separation $d$, obtained by the numerical method (symbols) and by the asymptotic expressions (curves) from Eqs. (15) and (16), for (a) $\mu=1.7$ and (b) $\mu=0.6$. The forces are plotted on a $\log _{10}$ scale and the values of the fitting parameters $p, q, \gamma$, and $\delta$ are given in each panel.
TABLE III. Fitting parameters $\eta_{i}$ and estimated variance $v$ for Eq. (17) in the V-GV case ( $n_{1}=1$ and $\left.n_{2}=2\right)$ for $\mu$ between 0.2 and 2.5.

\begin{tabular}{llcccc}
\hline \hline$\mu$ & \multicolumn{1}{c}{$\eta_{1}$} & $\eta_{2}$ & $\eta_{3}$ & $\eta_{4}$ & $v\left(\times 10^{-8}\right)$ \\
\hline 0.2 & $1.495 \times 10^{-9}$ & $6.073 \times 10^{-10}$ & 8.440 & -443.65 & 1.72 \\
0.3 & $2.347 \times 10^{-7}$ & $4.944 \times 10^{-8}$ & 6.929 & -58.765 & 16.2 \\
0.4 & $1.165 \times 10^{-6}$ & $1.69 \times 10^{-7}$ & 6.679 & -24.312 & 33.7 \\
0.5 & $2.302 \times 10^{-6}$ & $2.823 \times 10^{-7}$ & 6.628 & -19.433 & 22.6 \\
0.6 & $1.083 \times 10^{-5}$ & $1.230 \times 10^{-6}$ & 6.199 & -8.372 & 8.6 \\
0.7 & $6.775 \times 10^{-5}$ & $6.892 \times 10^{-6}$ & 5.681 & -1.661 & 2.6 \\
0.8 & $3.681 \times 10^{-4}$ & $3.283 \times 10^{-5}$ & 5.173 & 0.446 & 0.91 \\
0.9 & $1.54 \times 10^{-3}$ & $1.196 \times 10^{-4}$ & 4.706 & 0.942 & 0.41 \\
1.1 & $3.81 \times 10^{-3}$ & $3.847 \times 10^{-4}$ & 4.539 & 1.049 & 0.69 \\
1.2 & $5.98 \times 10^{-3}$ & $6.366 \times 10^{-4}$ & 4.423 & 1.095 & 6.0 \\
1.3 & $8.78 \times 10^{-3}$ & $1.01 \times 10^{-3}$ & 4.330 & 1.140 & 21.7 \\
1.4 & $1.237 \times 10^{-2}$ & $1.55 \times 10^{-3}$ & 4.248 & 1.183 & 54.0 \\
1.5 & $1.684 \times 10^{-2}$ & $2.3 \times 10^{-3}$ & 4.175 & 1.225 & 108.8 \\
1.6 & $2.177 \times 10^{-2}$ & $3.27 \times 10^{-3}$ & 4.148 & 1.265 & 196.0 \\
1.7 & $2.813 \times 10^{-2}$ & $4.57 \times 10^{-3}$ & 4.095 & 1.304 & 315.8 \\
1.8 & $3.561 \times 10^{-2}$ & $6.22 \times 10^{-3}$ & 4.048 & 1.342 & 474.8 \\
1.9 & $4.428 \times 10^{-2}$ & $8.28 \times 10^{-3}$ & 4.008 & 1.378 & 676.0 \\
2.0 & $5.421 \times 10^{-2}$ & $1.08 \times 10^{-2}$ & 3.973 & 1.414 & 676.0 \\
2.1 & $6.549 \times 10^{-2}$ & $1.384 \times 10^{-2}$ & 3.942 & 1.449 & 1218 \\
2.2 & $7.818 \times 10^{-2}$ & $1.747 \times 10^{-2}$ & 3.916 & 1.483 & 1564 \\
2.3 & $9.236 \times 10^{-2}$ & $2.174 \times 10^{-2}$ & 3.894 & 1.517 & 1962 \\
2.4 & 0.1081 & $2.671 \times 10^{-2}$ & 3.876 & 1.549 & 2410 \\
2.5 & 0.1255 & $3.245 \times 10^{-2}$ & 3.861 & 1.581 & 2911 \\
\hline \hline
\end{tabular}

well with the numerical results. This suggests that the fitting function given by Eq. (17) cannot only be used for the V-V, but also for the V-GV interaction force.

The four fitting parameters $\eta_{i}(i=1-4)$ found for each value of $\mu$ in the V-GV case are shown in Table III for $\mu$ from 0.2 to 2.5 . As in the $\mathrm{V}-\mathrm{V}$ case, the estimated variance $v$ increases for $\mu>1$, hence the offered function is expected to fail in the extreme type-II case. As an example, the V-GV interaction force for $\mu=1.7$ and 0.6 is shown in Fig. 7 as a function of the $\mathrm{V}-\mathrm{GV}$ separation $d$, along with the fitting curves given by Eq. (17) with the corresponding parameters in Table III. Although the estimated variances for these cases are smaller than $10^{-5}$, it can be seen in the log plot in Figs. 7(b) and 7 (c) that for small separation the fitting function is less accurate as compared to the $\mathrm{V}-\mathrm{V}$ case shown in Fig. 3, where the variances are lower than $10^{-7}$. Nevertheless, in the low $d$ region the force is small and consequently the deviation in the force will also be small.

The dependence of the fitting parameters on $\mu$ is shown in Fig. 8 (squares) for the case of a $n_{1}=1$ and $n_{2}=2 \mathrm{~V}-\mathrm{GV}$ interaction, where the fitting to the data is also shown (curves). Once more, the three different behaviors of $\eta_{i}$ as a function of $\mu$ are observed, with data fitted with different parameters $A_{i}, B_{i}$, and $C_{i}$, using Eqs. (18a)-(18d). The reason for the existence of three identifiable behaviors of the parameters $\eta_{i}$ as a function of $\mu$ is the same as in the V-V case. Moreover, for $\mu>1$ (type-II regime), we found $\eta_{4}(\mu) \approx \sqrt{\mu}$, similar to the $\mathrm{V}-\mathrm{V}$ case. The parameters $A_{i}, B_{i}$, and $C_{i}$ for the $\mathrm{V}-\mathrm{GV}$ case, for each range of $\mu$, are given in Table IV. 


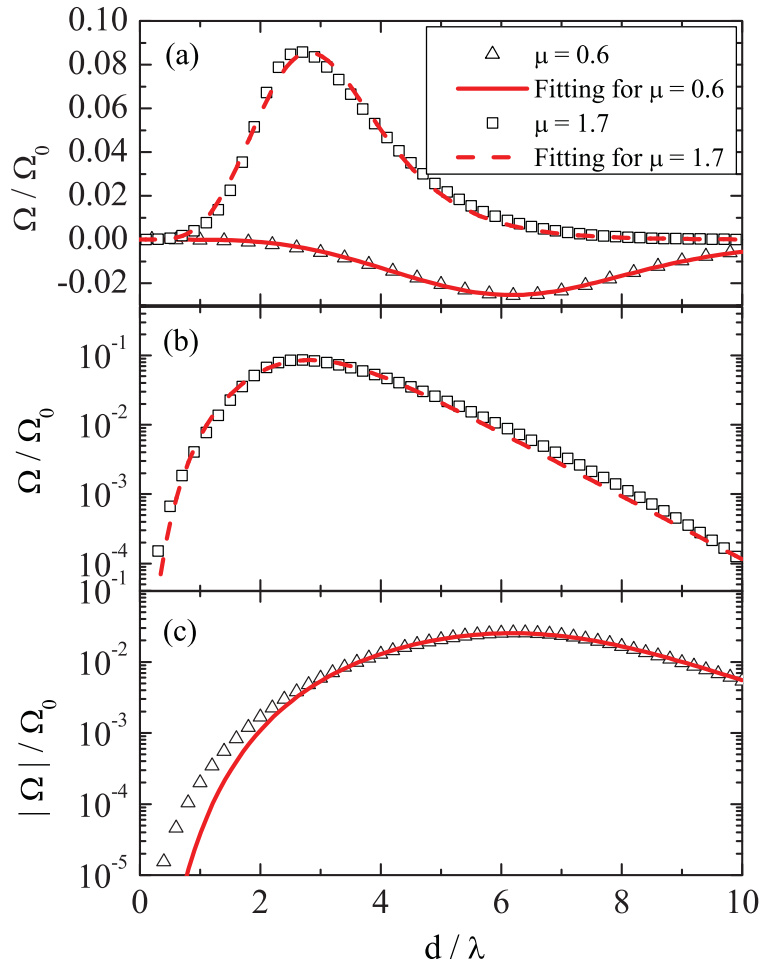

FIG. 7. (Color online) (a) Comparison between the V-GV interaction force as a function of the separation $d$ obtained by the numerical method (symbols) and by the fitting function (curves) given by Eq. (17). The solid (dashed) curves and the open triangles (squares) are the data for $\mu=0.6(1.7)$. The results for each $\mu$ are also plotted separately on a log scale: (b) $\mu=1.7$; (c) $\mu=0.6$.

\section{The three-vortex problem}

Up to now, we considered only the two-body interaction of vortices. In the study of the dynamics of many vortices, one generally considers the sum of pairwise interactions. In this sense, the force acting on vortex $i$ in a system with many vortices forming a certain configuration is given by ${ }^{32,33}$

$$
\vec{\Omega}_{i}=\sum_{j \neq i} \Omega\left(\left|\vec{r}_{i}-\vec{r}_{j}\right|\right) \hat{r}_{i, j},
$$

where $\vec{r}_{i}$ is the position of the vortex $i$ and $\hat{r}_{i, j}=\left(\vec{r}_{i}-\vec{r}_{j}\right) /$ $\left|\vec{r}_{i}-\vec{r}_{j}\right|$. In such a model, the $\mathrm{V}-\mathrm{V}$ interaction force is derived from the interaction potential between a pair of vortices, and is usually taken as $\Omega\left(\left|\vec{r}_{i}-\vec{r}_{j}\right|\right)=f_{0} K_{1}\left(\left|\overrightarrow{r_{i}}-\overrightarrow{r_{j}}\right| / \lambda\right)$, where $f_{0}$ is a constant. As mentioned in Sec. IV A, this corresponds to the extreme type-II situation $(\mu \gg 1)$. For intermediate values of $\mu$, one should consider both Bessel functions in Eq. (10), which is not convenient because, as mentioned in Sec. II, this expression diverges for small V-V separation and does not take into account either the vortex core deformations or the formation of giant vortices. Hence, using Eq. (17) for the vortex pair interaction force, with the parameters given by Table I or by Eqs. (18a)-(18d), would be an easy way to take these features into account and avoid the small separation divergence. Although this solves the problem in the standard simulations of vortex dynamics, which involve only pairwise interactions, here we will show when such an approach breaks

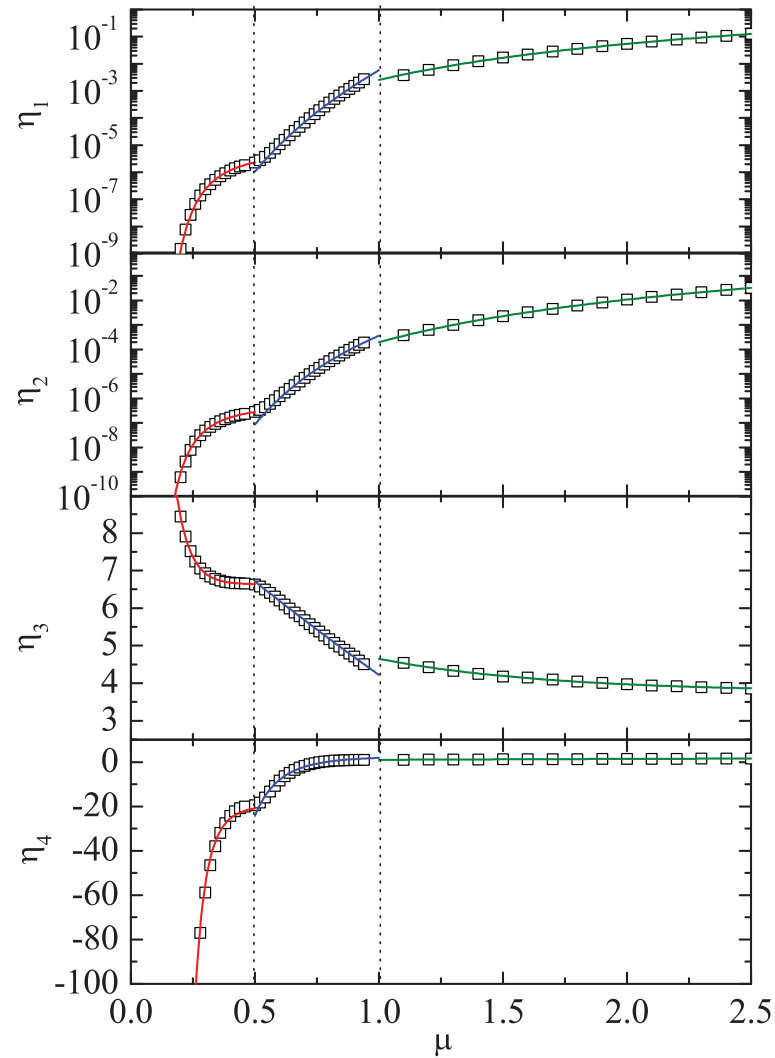

FIG. 8. (Color online) The fitting coefficients $\eta_{i=1-4}$ (symbols) in Eq. (17) as a function of $\mu=\sqrt{2} \kappa$ for the V-GV case. The curves present the $\eta_{i}(\mu)$ fittings given by Eqs. (18a)-(18d) for three different ranges, defined in the text as range $1(0<\mu<0.5)$, range $2(0.5<$ $\mu<1)$, and range $3(1<\mu)$.

down, and take the interaction between three vortices placed in the vertices of an equilateral triangle as an example.

Let us consider three vortices placed in the positions $r_{1}=(-d / 2,-\sqrt{3} d / 4), \quad r_{2}=(d / 2,-\sqrt{3} d / 4), \quad$ and $r_{3}=$ $(0, \sqrt{3} d / 4)$, forming a triangle of side $d$. The three-vortex ansatz is $\Psi=e^{i n_{1} \theta_{1}} e^{i n_{2} \theta_{2}} e^{i n_{3} \theta_{3}} f(x, y)$, where we control the vorticity $n_{1}, n_{2}$, and $n_{3}$ of each vortex. For the present example, we consider three singly quantized vortices $n_{1}=n_{2}=n_{3}=1$. Following the procedure described in Sec. III, we obtain the

TABLE IV. Fitting parameters in Eqs. (18a)-(18d) for the V-GV case $\left(n_{1}=1\right.$ and $\left.n_{2}=2\right)$ for three different ranges of $\mu$.

\begin{tabular}{lccc}
\hline \hline Parameter & $\mu<0.5$ & $0.5<\mu<1$ & $\mu>1$ \\
\hline$A_{1}$ & 61.948 & -0.9926 & -0.5785 \\
$B_{1}$ & -0.1937 & -693.484 & -14.199 \\
$C_{1}$ & -2.350 & $-1.779 \times 10^{-2}$ & -0.3514 \\
$A_{2}$ & 101.28 & -0.6458 & -0.5461 \\
$B_{2}$ & -0.1417 & -22.365 & -18.823 \\
$C_{2}$ & -2.374 & -0.4566 & -0.3461 \\
$A_{3}$ & 6.631 & -15.424 & 3.761 \\
$B_{3}$ & 67.184 & 24.938 & 3.748 \\
$C_{3}$ & -18.061 & -0.2385 & -1.442 \\
$A_{4}$ & -19.425 & 2.654 & 0 \\
$B_{4}$ & $-2.396 \times 10^{-2}$ & -0.7764 & 1 \\
$C_{4}$ & -6.075 & -5.113 & 0.5 \\
\hline \hline
\end{tabular}




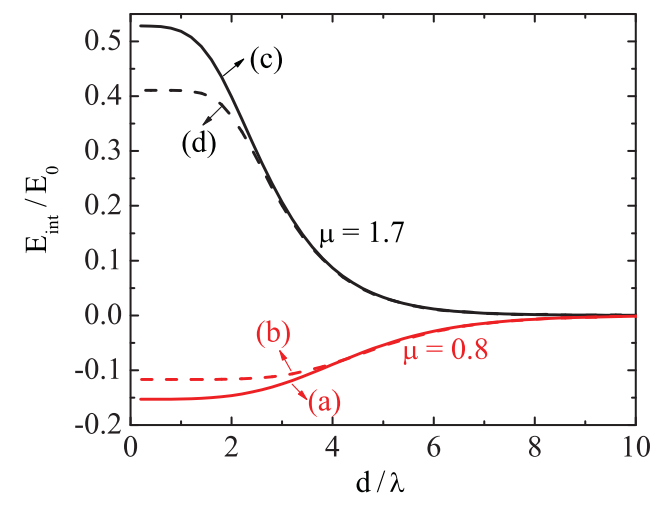

FIG. 9. (Color online) Interaction energy for three vortices placed in the vertices of an equilateral triangle as a function of its side $d$, obtained by the three vortices ansatz (dashed) and by considering only interactions between pairs (solid), i.e., $E_{\mathrm{int}}(d)=3 E_{\mathrm{int}}^{\text {pair }}(d)$, for $\mu=0.8$ and 1.7 .

Euler-Lagrange equations for the three-vortex problem, where the first equation is similar to Eq. (13a), but $\bar{X}$ and $\bar{Y}$ contain three terms,

$$
\bar{X}=\frac{x_{1}}{r_{1}^{2}}+\frac{x_{2}}{r_{2}^{2}}+\frac{x_{3}}{r_{3}^{2}}, \quad \bar{Y}=\frac{y_{1}}{r_{1}^{2}}+\frac{y_{2}}{r_{2}^{2}}+\frac{y_{3}}{r_{3}^{2}},
$$

and the second equation is

$$
\vec{\nabla} \times \vec{\nabla} \times \vec{A}+\left[\vec{A}-\frac{1}{r_{1}} \widehat{\theta_{1}}-\frac{1}{r_{2}} \widehat{\theta}_{2}-\frac{1}{r_{3}} \widehat{\theta_{3}}\right] f^{2}=0 .
$$

Similarly to the case of $\mathrm{V}-\mathrm{V}$ and $\mathrm{V}-\mathrm{GV}$ interactions, we solved the Euler-Lagrange equations for three vortices numerically by means of a finite-difference scheme and a relaxation method. The results obtained for the interaction energy as a function of the $\mathrm{V}-\mathrm{V}$ distance, or equivalently, the side $d$ of the triangle, are shown as dashed curves in Fig. 9 for two values of the GL parameter, $\mu=0.8$ (typeI) and 1.7 (type-II). As we consider the same distance $d$ between each pair of vortices forming the triangle, the standard procedure for the many-vortex problem, which considers only pair interactions, predicts an interaction energy $E_{\text {int }}(d)=$ $3 E_{\text {int }}^{\text {pair }}(d)$, where $E_{\text {int }}^{\text {pair }}(d)$ is the interaction energy for each $\mathrm{V}-\mathrm{V}$ pair. This energy is shown by the solid curves in Fig. 9, where good agreement with the results obtained from the three-vortex ansatz is observed only for larger separations $d$, whereas for smaller separations the energies predicted by the pair interaction model are clearly overestimated. This result is a manifestation of the importance of the vortex deformations for small $\mathrm{V}-\mathrm{V}$ separation: The pairs interaction model simply does not account for giant vortex deformations with three vortices. As a consequence, this model overestimates the energy. This is illustrated in Fig. 10, where the amplitude of the order parameter for the two [Figs. 10(a) and 10(c)] and three [Figs. 10(b) and 10(d)] interacting vortices is shown for $\mu=0.8$ (left-hand panels) and 1.7 (right-hand panels) at V-V separations $d=3.2 \lambda$ and $1.8 \lambda$, respectively. In the case of three vortices, we observe that each vortex is deformed toward the center of the vortex cluster. Such a deformation, which is found as the lowest-energy state of the three-vortex system,

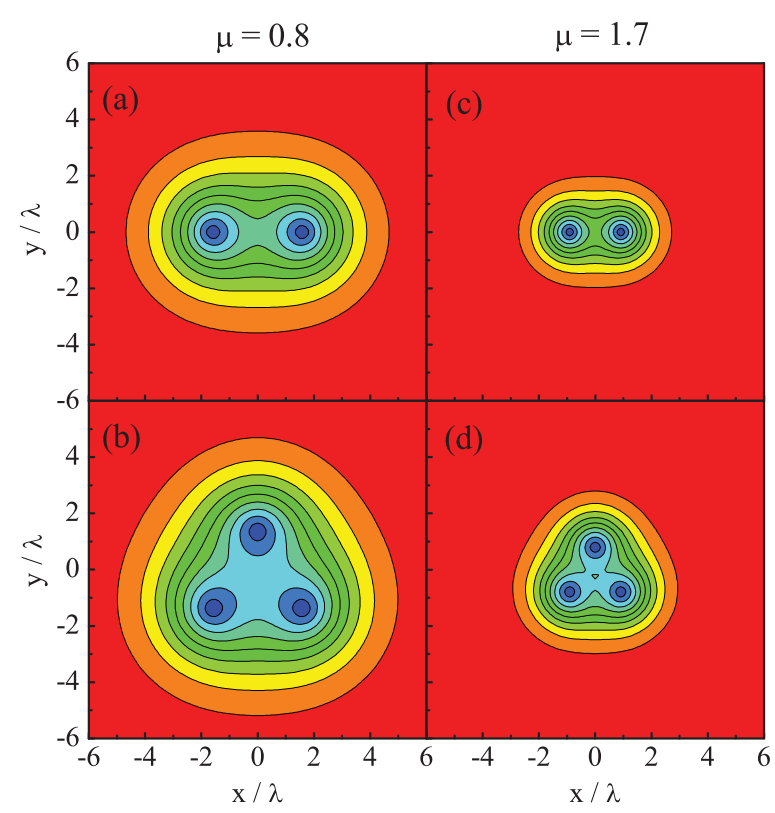

FIG. 10. (Color online) Amplitude of the order parameter for the cases indicated in Fig. 9, namely, for $\mu=0.8$ (1.7) and $d=3.2 \lambda$ $(1.8 \lambda)$, obtained for the two (a), (c) and three (b), (d) interacting vortices.

cannot be obtained by a model consisting only of interactions between pairs of vortices. Nonetheless, in the extreme type-II cases studied in the literature, ${ }^{32,33}$ the critical V-V separation $d_{c}$ where the vortices start to coalesce approaches zero (as demonstrated in the previous subsection) and the agreement between the results obtained by the pairwise model and by the three-vortex ansatz is expected to improve at even smaller separations.

In the many-vortex problem, the vortices can approach each other in a combinatorially large number of ways, and the study of three vortices in a triangular geometry presented in this subsection is a very specific case. Nevertheless, this example illustrates in a simple way that, apart from the extreme type-II case, an exact description of the many-vortex dynamics is a very difficult task. The pair potential, even when taking V-V deformations into account, still provides only an approximate description of the problem, as the deformations owing to the presence of all the other vortices are not included in the model. In this sense, the expressions proposed in the present work for the $\mathrm{V}-\mathrm{V}$ and $\mathrm{V}-\mathrm{GV}$ interaction forces provide an important improvement on the well-known expressions $\Omega\left(\left|\vec{r}_{i}-\vec{r}_{j}\right|\right)=$ $f_{0} K_{1}\left(\left|\vec{r}_{i}-\vec{r}_{j}\right| / \lambda\right)$ and Eq. (10), by including the deformations and the merger of vortices, but a MD study of many vortices using these expressions is still not an ideal description of a system with comparable length scales $\xi$ and $\lambda$.

\section{Vortex-antivortex interaction}

In the previous subsections, we showed that when two vortices or a vortex and a giant vortex are brought close to each other, they merge forming a single giant vortex state with vorticity $n=n_{1}+n_{2}$, and in the limit of small separation the $\mathrm{V}-\mathrm{V}$ or $\mathrm{V}-\mathrm{GV}$ forces are very weak. Conversely, as discussed in Sec. II, a vortex and an antivortex attract and annihilate, both in type-I and type-II superconductors. In what follows, 
the behavior of the force for the vortex-antivortex (V-AV) interaction as a function of the $\mathrm{V}-\mathrm{AV}$ separation is studied in greater detail.

Indeed, the $\mathrm{V}-\mathrm{AV}$ interaction is quite different from the interaction observed in the $\mathrm{V}-\mathrm{V}$ and $\mathrm{V}-\mathrm{GV}$ cases studied in previous subsections. The $\mathrm{V}-\mathrm{AV}$ interaction energy (a) and force (b) are shown in Fig. 11 for two values of the GL parameter, $\mu=0.6$ (triangles) and 1.7 (squares). As discussed previously in Sec. II, the $\mathrm{V}$-AV interaction is always attractive for any value of $\mu$. However, at some critical V-AV separation $d_{E}$, the solution with well-defined supercurrents around each vortex and antivortex ceases to be the lowest-energy state of the system. A solution with lower energy exhibits a strong suppression of the amplitude of the order parameter and supercurrent in the region between the vortex and the antivortex, and represents the ground state for small V-AV distances. A hysteresis is observed in the vicinity of the critical separation $d_{E}$, as shown in Fig. 11(a). These results resemble those obtained by Priour and Fertig ${ }^{34}$ in the case of a vortex placed close to an artificial defect. A suppressed amplitude
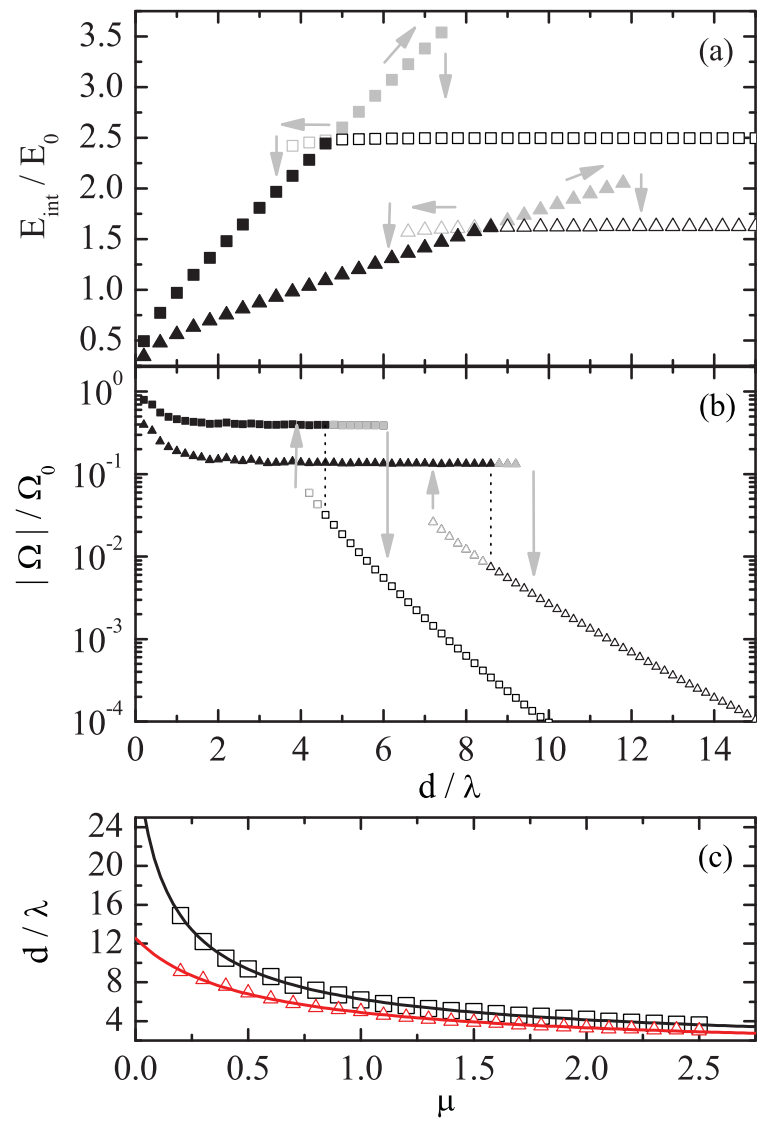

FIG. 11. (Color online) V-AV interaction (a) energy and (b) force (absolute value), as a function of the separation $d$, for $\mu=0.6$ (triangles) and 1.7 (squares). The full (open) symbols are the results obtained in the numerical relaxation procedure by gradually increasing (decreasing) $d$ from 0 to $15 \lambda$ (from $15 \lambda$ to 0 ). A hysteresis is observed around a critical separation $d_{E}$, as indicated by the arrows, and the solution represented by open symbols is stable only for $d>d_{A}$. (c) Numerically obtained critical separations $d_{E}$ (squares) and $d_{A}$ (triangles) as a function of the GL parameter $\mu$, along with their fitting functions (curve). of the order parameter is also observed by Sardella et al. ${ }^{35}$ in the dynamics of $\mathrm{V}-\mathrm{AV}$ annihilation in a square mesoscopic superconducting cylinder, for small V-AV separation. The absolute value of the force is shown in Fig. 11(b) on a $\log _{10}$ scale, where two different behaviors, for separations $d$ smaller and larger than $d_{E}$, are clearly observed.

The dependence of the numerically obtained critical separation $d_{E}$ for the $\mathrm{V}-\mathrm{AV}$ interaction on the GL parameter $\mu$ is illustrated as the squares in Fig. 11(c), and can be fitted to a function similar to the one used for the critical separations in the V-V and V-GV cases, given by $d_{E}=0.337+31.249(1+$ $10.264 \mu)^{-0.6855}$ (with an estimated variance $v \approx 0.4 \%$ ), which is shown as a solid curve in Fig. 10(c). The difference is that the $\mu \rightarrow \infty$ limit is now finite, while previously it was zero. Although the solution with well-defined supercurrents around each vortex and antivortex is not the lowest-energy state for $d<d_{E}$, it is still a stable state in the vicinity of this point, and becomes unstable only for $d<d_{A}$. The dependence of $d_{A}$ on the GL parameter $\mu$ is shown by the triangles in Fig. 11(c) and can be fitted by $d_{A}=0.337+12.222(1+2.461 \mu)^{-0.7931}$ (with an estimated variance $v \approx 0.6 \%$ ).

Figure 12 shows the distribution of the supercurrent $\vec{J}=$ $\vec{\nabla} \times \vec{\nabla} \times \vec{A}$ and the amplitude of the order parameter along the direction of the V-AV approach ( $y=0$ axis) for different values of the $\mathrm{V}-\mathrm{AV}$ separation. The critical separations for $\mu=0.6$ and 1.7 are $d_{E}=8.6 \lambda$ and $4.5 \lambda$, respectively, and the values of $\mathrm{V}$-AV separation in Fig. 12 are chosen as $d=$ $9.2 \lambda>d_{E}$ for $\mu=0.6$ and $5.2 \lambda>d_{E}$ for $\mu=1.7$. Notice that for each of these separations, we can find two solutions
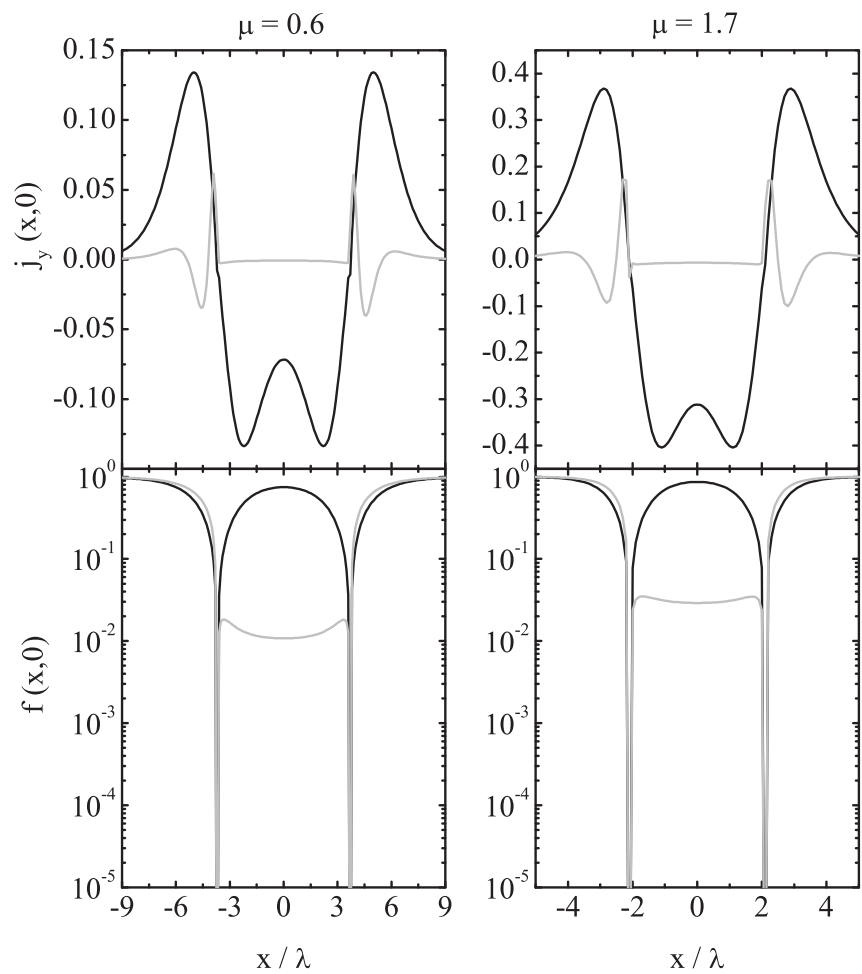

FIG. 12. Supercurrent (top) and amplitude of the order parameter (bottom) along the direction of the V-AV approach $x$, for the V-AV separation $d=9.2 \lambda(5.2 \lambda)$, for $\mu=0.6$ (1.7). Black (gray) curves refer to the states represented by open (full) symbols in Fig. 11(b). 
with different energies. The black (gray) curves in Fig. 12 are related to the open (full) symbols in Fig. 11(b). When the $\mathrm{V}$-AV separation is large, the currents around the vortex and the antivortex present well-defined peaks at some distance, which depends on $\mu$. As the vortex and antivortex are placed closer, their supercurrents superimpose in the region between them, as observed in the black curves in Fig. 12 (top). The black curves in Fig. 12 (bottom) show that the amplitude of the order parameter in these solutions has zeros at each vortex and antivortex position and reaches $\approx 1$ in the region between them. In the vicinity of $d_{E}$, for V-AV separation $d>d_{E}$, there is a higher-energy state [see the full gray symbols in Fig. 11(a)] with strongly suppressed supercurrents and an amplitude of the order parameter in the region between the vortex and antivortex, which is shown by the gray curves in Fig. 12 . For $d<d_{E}$, the solution represented by black lines in Fig. 12 is no longer the lowest-energy state, as shown in Fig. 11(a), and becomes unstable as the $\mathrm{V}-\mathrm{AV}$ separation is reduced at $d<d_{A}$, while the solution with suppressed current and order parameter between the vortex and antivortex, shown by the gray curves, becomes the lowest-energy state for $d<d_{E}$ and the only stable solution for $d<d_{A}$.

The suppressed order parameter in the region between vortices observed in the only stable solution for $d<d_{A}$ suggests that a vortex and an antivortex cannot coexist at these distances, unless somehow pinned, in which case this string solution is formed. This is reasonable, because at short distances the fields of the vortex and the antivortex compensate each other, and the flux quantization as an essential property of a(n) (anti)vortex is lost. Notice that this is different from the case of two merging vortices, which can coexist at short distances, deform, and interact as described in previous sections, because the flux quantization of the $\mathrm{V}-\mathrm{V}$ pair is preserved even at small $\mathrm{V}-\mathrm{V}$ separations. The string formation goes beyond simulations of $\mathrm{V}-\mathrm{AV}$ dynamics, because in this case the V-AV pair is no longer well defined by their surrounding supercurrent and order parameters. For MD studies of the V-AV motion, one should consider the critical separation $d_{A}$ as the separation where the V-AV pair annihilates (see, e.g., Ref. 36).

Owing to the peculiar behavior found for the $\mathrm{V}$ - $\mathrm{AV}$ force as a function of the separation $d$, which is discontinuous at $d_{E}$, it is not possible to find a single fitting function describing the force for both the $d>d_{E}$ and $d<d_{E}$ regimes, as we were able to do for the V-V and V-GV forces. On the other hand, as discussed in the previous section, the $\mathrm{V}-\mathrm{AV}$ interaction force at large distances $d$ can be described by a combination of Bessel functions, given by Eq. (10), which can be rewritten as

$$
\Omega(d)=-\Delta_{1} K_{1}(d)-\Delta_{2} K_{1}(\mu d),
$$

where $\Delta_{1}$ and $\Delta_{2}$ are fitting parameters. We have fitted our numerically obtained $\mathrm{V}-\mathrm{AV}$ interaction force for $d>d_{E}$ using Eq. (21), and a list of fitting parameters for the GL parameter $\mu$ ranging from 0.3 to 2.5 is given in Table $V$. A list of such fitting parameters can also be found in Ref. 7, where the relation between our fitting parameters and the parameters $q$ and $m$ of the cited work is $\Delta_{1}=m^{2} / 2 \pi^{2}$ and $\Delta_{2}=\mu q^{2} / 2 \pi^{2}$. Following the same procedure of previous subsections, we
TABLE V. Fitting parameters $\Delta_{i}$ and estimated variance $v$ for Eq. (21) in the V-AV case $\left(n_{1}=1\right.$ and $\left.n_{2}=-1\right)$ for $d>d_{E}$.

\begin{tabular}{lrcl}
\hline \hline$\mu$ & $\Delta_{1}$ & $\Delta_{2}$ & $v\left(\times 10^{-9}\right)$ \\
\hline 0.3 & 156.948 & 0.4203 & 0.918 \\
0.4 & 67.315 & 0.7419 & 0.10 \\
0.5 & 31.064 & 1.173 & 0.098 \\
0.6 & 19.070 & 1.719 & 0.18 \\
0.7 & 14.401 & 2.340 & 0.342 \\
0.8 & 11.990 & 2.925 & 0.56 \\
0.9 & 6.499 & 5.060 & 3.06 \\
1.0 & 6.357 & 6.357 & 5.01 \\
1.1 & 5.320 & 9.170 & 5.0 \\
1.2 & 4.159 & 14.269 & 1.21 \\
1.3 & 4.254 & 16.759 & 1.21 \\
1.4 & 4.125 & 20.640 & 1.10 \\
1.5 & 4.039 & 23.58 & 0.75 \\
1.6 & 3.775 & 33.662 & 1.20 \\
1.7 & 3.632 & 43.173 & 1.40 \\
1.8 & 3.542 & 51.251 & 1.03 \\
1.9 & 3.422 & 66.755 & 1.23 \\
2.0 & 3.315 & 87.448 & 1.38 \\
2.1 & 3.226 & 113.229 & 1.54 \\
2.2 & 3.162 & 138.472 & 1.25 \\
2.3 & 3.101 & 170.634 & 1.08 \\
2.4 & 3.037 & 220.086 & 1.13 \\
2.5 & 2.983 & 277.742 & 1.08 \\
\hline \hline
\end{tabular}

propose fitting functions for these parameters as a function of the GL parameter $\mu$,

and

$$
\Delta_{1}(\mu)=2.879+3.415 \mu^{-3.166}
$$

$$
\Delta_{2}(\mu)=\mu\left(-0.2258+1.044 e^{1.866 \mu}\right),
$$

which are plotted as solid lines in Fig. 13 along with the data of Table V (symbols).

Notice that for the V-AV interaction, we did not find different behaviors in different ranges of $\mu$, as observed for the $\mathrm{V}-\mathrm{V}$ and $\mathrm{V}-\mathrm{GV}$ cases, because the V-AV interaction is

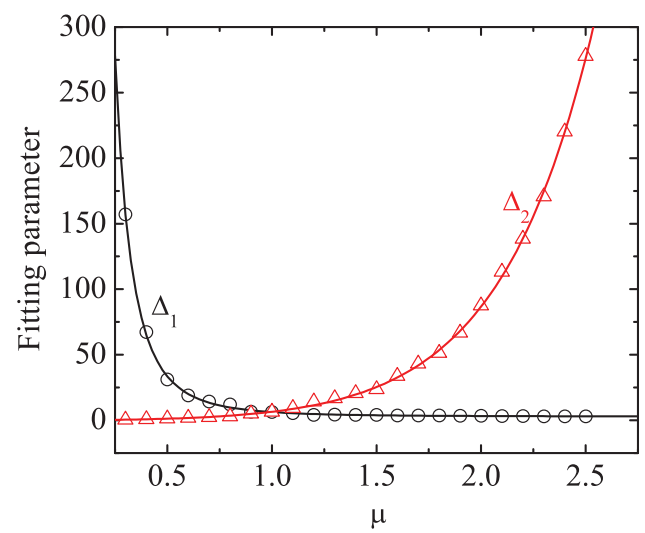

FIG. 13. (Color online) Fitting functions (curves) for the parameters $\Delta_{1}$ and $\Delta_{2}$ in Eq. (21), for the V-AV interaction force at $d>d_{E}$, as a function of the GL parameter $\mu=\sqrt{2} \kappa$. The data from Table V are shown as symbols for comparison. 
always attractive and becomes only stronger as $\mu$ increases from zero, instead of exhibiting zero force at $\mu=1$ and becoming repulsive for $\mu>1$, as observed for $\mathrm{V}-\mathrm{V}$ and $\mathrm{V}-\mathrm{GV}$ interactions. Therefore, substituting Eqs. (22) into Eq. (21) yields a single expression,

$$
\begin{aligned}
\Omega(d)= & -\left(2.879+3.415 \mu^{-3.166}\right) K_{1}(d) \\
& +\left(0.2258-1.044 e^{1.866 \mu}\right) \mu K_{1}(\mu d),
\end{aligned}
$$

which is expected to provide an accurate description of the $\mathrm{V}-\mathrm{AV}$ interaction force, at separations $d>d_{E}$, for any value of $\mu$.

\section{CONCLUSIONS}

We presented a theoretical study of the interaction between vortices in bulk superconductors within the GL theory. An analytical study of the asymptotic behavior of the V-V interaction shows that a combination of first-order modified Bessel functions of the second kind describes the behavior of the numerically obtained forces for large $\mathrm{V}-\mathrm{V}$ separation. At small distances, the fitting curves for $\mathrm{V}-\mathrm{V}$ and $\mathrm{V}-\mathrm{GV}$ interactions show that the force in this region behaves as a power function of the separation between vortices. We proposed a fitting function that combines both limiting behaviors, namely, the power law for small distances and the modified Bessel function behavior for large distances. This function, given by Eq. (17), gives a fairly accurate fitting of the interaction force for any value of $\mu$, even in the type-I regime. It depends on four fitting parameters, which can be obtained for any value of $\mu$ either by interpolating our data presented in Table I (Table III), for $\mathrm{V}-\mathrm{V}$ (V-GV) interactions, or by using Eqs. (18a)-(18d) with the parameters presented in Table II (Table IV).

Our analytical study of the V-AV interaction shows that the $\mathrm{V}-\mathrm{AV}$ interaction force is attractive for any value of the GL parameter $\mu$, which is confirmed by our numerical results and contradicts the conjecture proposed in previous works, ${ }^{19-21}$ which implies that the $\mathrm{V}-\mathrm{AV}$ interaction force is repulsive for $\mu<1$ (type-I). For large $\mathrm{V}-\mathrm{AV}$ separation $d$, the interaction force decays with $d$ as a combination of modified Bessel functions. However, for $d$ smaller than a critical separation $d_{E}$, the conventional $\mathrm{V}-\mathrm{AV}$ pair is no longer the lowest-energy state. Instead, the lowest-energy state exhibits a strong suppression of the supercurrent and amplitude of the order parameter in the region between the vortex and antivortex, which results in a different behavior of the force as a function of $d$ in this case and, as a consequence, the $\mathrm{V}-\mathrm{AV}$ interaction force is discontinuous at $d_{E}$. Furthermore, the conventional $\mathrm{V}$-AV pair becomes unstable for $d$ lower than the separation $d_{A}$, which is interpreted as the $\mathrm{V}-\mathrm{AV}$ annihilation point. We fitted the interaction force for V-AV separations $d>d_{E}$ by Eq. (21) and proposed an approximate analytical expression for the $\mathrm{V}$-AV interaction force at these separations, given by Eq. (23), which is valid for any value of $\mu$.

The fitting functions for the V-V, V-GV, and V-AV force given in this work will be useful, for instance, for the study of bulk and mesoscopic systems consisting of many vortices using MD techniques. We nevertheless remark that, although deformations are taken into account in the interaction force between two vortices in this work, the deformations in a many-vortices system are expected to be more complex. Hence, the MD study of many vortices, even with the improved expressions for the interaction force provided in this paper, is still an approximate description of the system. As a method, the derivation and handling of the differential equations describing the interaction between vortices presented in this work can be further adapted to describe such interactions in, e.g., two-band superconductors, or hybrid systems comprising different superconducting materials.

\section{ACKNOWLEDGMENTS}

Discussions with J. S. Andrade Jr. and A. A. Moreira are gratefully acknowledged. This work was financially supported by CNPq, under Contract No. NanoBioEstruturas 555183/2005-0, PRONEX/FUNCAP, CAPES, the Bilateral programme between Flanders and Brazil, the collaborative project CNPq-FWO-Vl, the Belgian Science Policy (IAP), and the Flemish Science Foundation (FWO-Vl).

\footnotetext{
*andrey@ fisica.ufc.br

$\dagger$ gil@fisica.ufc.br

${ }^{1}$ L. Kramer, Phys. Rev. B 3, 3821 (1971).

${ }^{2}$ L. Jacobs and C. Rebbi, Phys. Rev. B 19, 4486 (1979).

${ }^{3}$ V. A. Schweigert and F. M. Peeters, Phys. Rev. B 57, 13817 (1998).

${ }^{4}$ A. Kanda, B. J. Baelus, F. M. Peeters, K. Kadowaki, and Y. Ootuka, Phys. Rev. Lett. 93, 257002 (2004).

${ }^{5}$ D. S. Golubović, M. V. Milošević, F. M. Peeters, and V. V. Moshchalkov, Phys. Rev. B 71, 180502(R) (2005).

${ }^{6}$ E. H. Brandt, Phys. Rev. B 34, 6514 (1986).

${ }^{7}$ J. M. Speight, Phys. Rev. D 55, 3830 (1997).

${ }^{8}$ R. MacKenzie, M.-A. Vachon, and U. F. Wichoski, Phys. Rev. D 67, 105024 (2003).

${ }^{9}$ L. M. A. Bettencourt and R. J. Rivers, Phys. Rev. D 51, 1842 (1995).

${ }^{10}$ F. Mohamed, M. Troyer, G. Blatter, and I. Luk'yanchuk, Phys. Rev. B 65, 224504 (2002).

${ }^{11}$ A. D. Hernández and A. López, Phys. Rev. B 77, 144506 (2008).

${ }^{12}$ J. R. Clem, J. Low Temp. Phys. 18, 427 (1975).
}

${ }^{13}$ R. Auzzi, M. Eto, and W. Vinci, J. High Energy Phys. 2, 100 (2008).

${ }^{14}$ E. Babaev and M. Speight, Phys. Rev. B 72, 180502(R) (2005).

${ }^{15}$ R. Geurts, M. V. Milošević, and F. M. Peeters, Phys. Rev. B 81, 214514 (2010)

${ }^{16}$ D. R. Tilley and J. Tilley, Superfluidity and Superconductivity (IOP, London, 1990).

${ }^{17}$ E. Myers, C. Rebbi, and Richard Strilka, Phys. Rev. D 45, 1355 (1992).

${ }^{18}$ M. Gleiser and J. Thorarinson, Phys. Rev. D 76, 041701 (2007).

${ }^{19}$ V. R. Misko, V. M. Fomin, J. T. Devreese, and V. V. Moshchalkov, Phys. Rev. Lett. 90, 147003 (2003).

${ }^{20}$ V. R. Misko, V. M. Fomin, J. T. Devreese, and V. V. Moshchalkov, Phys. C 404, 251 (2004).

${ }^{21}$ G. Teniers, V. V. Moshchalkov, L. F. Chibotaru, and A. Ceulemans, Phys. B 329-333, 1340 (2003).

${ }^{22}$ G. R. Berdiyorov, M. V. Milošević, and F. M. Peeters, Phys. Rev. Lett. 96, 207001 (2006). 
${ }^{23}$ R. Geurts, M. V. Milošević, and F. M. Peeters, Phys. Rev. Lett. 97, 137002 (2006); Phys. Rev. B 75, 184511 (2007).

${ }^{24}$ E. B. Bogomol'nyi, Sov. J. Nucl. Phys. 24(4), 449 (1976).

${ }^{25}$ T. M. Samols, Commun. Math. Phys. 145, 149 (1992).

${ }^{26}$ William H. Press, Brian P. Flannery, Saul A. Teukolsky, and William T. Vetterling, Numerical Recipes in Fortran 77, 2nd ed. (Cambridge University Press, Cambridge, UK, 1992).

${ }^{27}$ A perfect fitting function gives $v \rightarrow 0$.

${ }^{28}$ J. Pearl, Appl. Phys. Lett. 5, 65 (1964).

${ }^{29}$ E. H. Brandt, Phys. Rev. B 79, 134526 (2009).

${ }^{30}$ S. Zapperi, A. A. Moreira, and J. S. Andrade Jr., Phys. Rev. Lett. 86, 3622 (2001).
${ }^{31}$ E. H. Brandt, Rep. Prog. Phys. 58, 1465 (1995).

${ }^{32}$ C. Reichhardt, C. J. Olson, and F. Nori, Phys. Rev. Lett. 78, 2648 (1997).

${ }^{33}$ W. V. Pogosov, H. J. Zhao, V. R. Misko, and F. M. Peeters, Phys. Rev. B 81, 024513 (2010).

${ }^{34}$ D. J. Priour Jr. and H. A. Fertig, Phys. Rev. B 67, 054504 (2003).

${ }^{35}$ E. Sardella, P. N. Lisboa Filho, C. C. de Souza Silva, L. R. Eulalio Cabral, and W. Aires Ortiz, Phys. Rev. B 80, 012506 (2009).

${ }^{36}$ C. L. S. Lima and C. C. de Souza Silva, Phys. Rev. B 80, 054514 (2009). 